\title{
A PHASE-FIELD MODEL FOR COMPLIANCE SHAPE OPTIMIZATION IN NONLINEAR ELASTICITY
}

\author{
Patrick Penzler ${ }^{1}$, Martin RumpF ${ }^{1}$ And Benedikt Wirth ${ }^{1}$
}

\begin{abstract}
Shape optimization of mechanical devices is investigated in the context of large, geometrically strongly nonlinear deformations and nonlinear hyperelastic constitutive laws. A weighted sum of the structure compliance, its weight, and its surface area are minimized. The resulting nonlinear elastic optimization problem differs significantly from classical shape optimization in linearized elasticity. Indeed, there exist different definitions for the compliance: the change in potential energy of the surface load, the stored elastic deformation energy, and the dissipation associated with the deformation. Furthermore, elastically optimal deformations are no longer unique so that one has to choose the minimizing elastic deformation for which the cost functional should be minimized, and this complicates the mathematical analysis. Additionally, along with the non-uniqueness, buckling instabilities can appear, and the compliance functional may jump as the global equilibrium deformation switches between different bluckling modes. This is associated with a possible non-existence of optimal shapes in a worst-case scenario. In this paper the sharp-interface description of shapes is relaxed via an Allen-Cahn or Modica-Mortola type phase-field model, and soft material instead of void is considered outside the actual elastic object. An existence result for optimal shapes in the phase field as well as in the sharp-interface model is established, and the model behavior for decreasing phase-field interface width is investigated in terms of $\Gamma$-convergence. Computational results are based on a nested optimization with a trust-region method as the inner minimization for the equilibrium deformation and a quasi-Newton method as the outer minimization of the actual objective functional. Furthermore, a multi-scale relaxation approach with respect to the spatial resolution and the phase-field parameter is applied. Various computational studies underline the theoretical observations.
\end{abstract}

Mathematics Subject Classification. 49Q10, 74P05, 49J20.

Received March 27, 2010.

Published online December 23, 2010.

\section{INTRODUCTION}

In this paper we investigate shape optimization in the context of nonlinear elastic constitutive laws for the underlying objects, where an object is defined as an object domain $\mathcal{O} \subset \Omega$ for some open connected set $\Omega \subset \mathbb{R}^{d}$. We are in particular interested in geometrically strongly nonlinear deformations $\phi: \mathcal{O} \rightarrow \mathbb{R}^{d}$ which minimize a certain hyperelastic energy. As hyperelastic energies, we consider polyconvex energy functionals $\int_{\Omega} \chi_{\mathcal{O}} W(\mathcal{D} \phi) \mathrm{d} x$, where the integrand $W$ depends on the principal invariants of the Cauchy-Green strain tensor

Keywords and phrases. Shape optimization, nonlinear elasticity, phase-field model, buckling deformations, $\Gamma$-convergence.

1 Institute for Numerical Simulation, Bonn University, Endenicher Allee 60, 53115 Bonn, Germany.

benedikt.wirth@ins.uni-bonn.de 
$\mathcal{D} \phi^{T} \mathcal{D} \phi$ and its evaluation is restricted to the actual elastic domain $\mathcal{O}$ (with characteristic function $\chi_{\mathcal{O}}$ ). For these functionals a nowadays already classical existence theory has been established $[11,12,18]$ and an effective minimization is also computationally feasible. With respect to the actual shape optimization of the shape $\mathcal{O}$, we aim at minimizing a compliance cost functional $\mathcal{J}[\phi, \mathcal{O}]$, to be evaluated for $\phi=\phi[\mathcal{O}]$ being an equilibrium deformation of $\mathcal{O}$ and thus a minimizer of the free energy. This type of compliance optimization has applications in engineering, where mechanical devices or structural components have to be developed that optimally balance material consumption or component weight with the component stiffness or rigidity. Since the compliance is related to the energy absorbed by the mechanical structure, minimizing the compliance is also related to reducing the risk of material failure. Nonlinear elasticity has a strong impact on the behavior of the optimization problem. Indeed, there exist several possible definitions for the compliance which are all equivalent in linearized elasticity but now in the context of nonlinear elasticity result in significantly different optimization problems. We may consider the change in potential energy of the surface load, the stored elastic deformation energy, or the dissipation associated with the deformation. The deformation induced by the surface load is in general no longer unique, which complicates the mathematical analysis. Buckling instabilities may appear, in which structures such as compressed beams can bend to either side, thereby producing non-uniqueness. Since different buckling deformations generally correspond to different compliance values, one may experience that the compliance suddenly jumps up during shape optimization as the global equilibrium deformation switches from one buckling deformation to another one. This phenomenon may result in non-existence of optimal shapes if we always pick the worst case from the set of all equilibrium deformations. Furthermore, in nonlinear elasticity, reversing the surface load (changing its sign) in general influences the mechanical response. As a consequence, shape optimization problems that yield symmetric shapes in linearized elasticity now result in asymmetric shapes.Finally, the use of nonlinear elasticity also poses numerical challenges. We typically observe rather large, geometrically strongly nonlinear deformations. Their computation requires robust numerical minimization methods that reliably detect local rotations and bypass saddle points which frequently appear between two buckling deformations.

To render the optimization problem computationally feasible we replace the void outside the actual elastic object $\mathcal{O}$ by a (some orders of magnitude) softer material. Indeed, we take into account the energy functional $\int_{\Omega}\left((1-\delta) \chi_{\mathcal{O}}+\delta\right) W(\mathcal{D} \phi) \mathrm{d} x$ for a small positive constant $\delta$. Furthermore, we propose a double well phase-field model of Allen-Cahn [8] or Modica-Mortola [27] type for an implicit description of the elastic object with a diffusive interface. Indeed, we describe elastic shapes by a phase-field function $v: \Omega \rightarrow \mathbb{R}$ and take into account an energy $\int_{\Omega} \varepsilon|\nabla v|^{2}+\frac{1}{\varepsilon} \Psi(v) \mathrm{d} x$, where $\Psi(v)=\frac{9}{16}\left(v^{2}-1\right)^{2}$ is a double well potential with minima at $v=-1$ and $v=1$, representing the two material phases $\mathcal{O}$ and $\Omega \backslash \overline{\mathcal{O}}$. For this model, we prove the existence of optimal, shape-encoding phase fields and study the model behavior for decreasing parameter $\varepsilon$, which describes the width of the diffusive interface, in terms of $\Gamma$-convergence. Furthermore, a variety of $2 \mathrm{D}$ numerical examples enables a detailed discussion of the impact of the nonlinear elasticity model on the shape optimization problem.

Overall, this article focuses on three issues. The first is an analytical examination of the compliance minimization problem in nonlinear elasticity. We will show that optimal shapes do exist if in case of multiple equilibrium deformations we only consider a certain, "optimal" deformation. Otherwise - in particular in a worst case scenario - we cannot expect the existence of optimal shapes (except for particular objective functionals). This seems to be a quite general phenomenon associated with non-uniquely solvable optimization constraints. The second focus lies on the examination of a phase-field approximation to the compliance minimization problem. Here, too, we obtain the same existence result as for the sharp interface case. However, for general objective functionals we do not obtain the $\Gamma$-convergence of the phase-field model to the sharp interface problem. This again is due to the above non-uniqueness phenomenon or general nature of pde-constrained optimization and thus is already interesting in itself. Of course, this renders the phase-field approximation questionable since the optimal phase field is not guaranteed to converge against an optimal shape for decreasing interface width. Nevertheless, due to the validity of a $\Gamma$ - lim inf inequality, the phase field will at least converge against a comparatively good shape, which makes the approximation sensible for engineering purposes. Finally, we intend to prove the feasibility of the approach by numerical simulations. 
The organization of the paper is as follows. Section 2 presents an overview over the related shape optimization literature. After presenting the optimization problem and its phase-field approximation in Section 3 we will briefly examine the nature of nonlinear elasticity in the compliance minimization context in Section 4 . The existence of minimizers and the sharp-interface limit of the phase-field model are studied in Section 5 before presenting the implementation in Section 6 and finally showing a few experiments in Section 7.

\section{RELATED WORK}

In shape optimization frequently not only the geometry of the shape contour $\partial \mathcal{O}$ of an elastic mechanical device $\mathcal{O}$ is of interest, but also the topology of $\mathcal{O}$ is subject to optimization. Typically, one considers as underlying state problem the system of equations of linearized elasticity,

$$
\operatorname{div} \sigma=0
$$

on $\mathcal{O}$, where the Cauchy stress $\sigma$ is given by $\mathbf{C} \epsilon[u]=\frac{1}{2} \mathbf{C}\left(\mathcal{D} u+\mathcal{D} u^{\mathrm{T}}\right)$ for the fourth-order elasticity tensor $\mathbf{C}$ and the displacement $u$. As boundary conditions, one might impose Dirichlet conditions $u=0$ ona fixed region $\Gamma_{D} \subset \partial \mathcal{O}$, Neumann boundary conditions $\sigma \nu=F$ ona fixed region $\Gamma_{N} \subset \partial \mathcal{O}$ with $\Gamma_{D} \cap \Gamma_{N}=\emptyset$ for a prescribed surface load $F$, and zero surface force boundary conditions $\sigma \nu=0$ on the remaining boundary $\partial \mathcal{O} \backslash\left(\Gamma_{D} \cup \Gamma_{N}\right)$. With respect to the modeling, $\Gamma_{D}$ and $\Gamma_{N}$ are usually a priori fixed parts of the boundary, whereas the remaining boundary is subject to the actual shape optimization. For the sake of simplicity, we ignore volume forces here. The range of usual objective functionals $\mathcal{J}[u, \mathcal{O}]$ is relatively diverse. The mechanical work of the load, the so-called compliance $\mathcal{C}=\frac{1}{2} \int_{\Gamma_{N}} F \cdot u \mathrm{~d} a$, is very popular $[2-5,24,32-35]$ since it equals the energy to be absorbed by the elastic structure. A related choice is the $L^{2}$-norm of the internal stresses $[2,4,5], \int_{\mathcal{O}}\|\sigma\|_{F}^{2} \mathrm{~d} x$. If a specific displacement $u_{0}$ is to be reproduced, then the $L^{2}$-distance $\int_{\Omega}\left|u-u_{0}\right|^{2} \mathrm{~d} x$ serves as the appropriate objective functional [4]. Other possibilities include functionals depending on the shape eigenfrequencies or the compliance for design-dependent loads $[6,13,30]$. Typically, the optimization problem is complemented by a volume constraint for $\mathcal{O}$ (otherwise, especially for compliance minimization, $\mathcal{O}=\Omega$ would be optimal). An equality constraint $|\mathcal{O}|=V$ is either ensured by a Cahn-Hilliard-type $H^{-1}$-gradient flow [37] or a Lagrangemultiplier ansatz $[6,13,25]$. A quadratic penalty term or an augmented Lagrange method is employed in [35]. An inequality constraint $|\mathcal{O}| \leq V$ is implemented in [24,33,34], using a Lagrange multiplier. Chambolle [16] exploits the monotonicity of the compliance $\mathcal{C}$ (in the sense $\mathcal{C}\left(\mathcal{O}_{1}\right) \geq \mathcal{C}\left(\mathcal{O}_{2}\right)$ for $\left.\mathcal{O}_{1} \subset \mathcal{O}_{2}\right)$ to replace the equality by an inequality constraint. Finally, the volume may just be added as a penalty functional $\nu|\mathcal{O}|$ for some parameter $\nu[2]$.

The above class of shape optimization problems is generically ill-posed since microstructures tend to form, which are associated with a weak but not strong convergence of the characteristic functions $\chi_{\mathcal{O}_{i}}$ along a minimizing sequence $\left(\mathcal{O}_{i}\right)_{i=1, \ldots}$. In particular, rank- $d$ sequential laminates with the lamination directions aligned with the stress eigendirections are known to be optimal for compliance minimization [3]. The above ill-posedness calls for regularization, for which there are several possibilities. A widespread approach is to penalize the shape perimeter by adding a term $\eta \mathcal{H}^{d-1}(\partial \mathcal{O})$ to the objective functional, which (if the void is replaced by some weak material) also results in existence of optimal shapes as studied in [9] for a scalar problem. An alternative consists in the relaxation of the problem: The set of admissible shapes can be extended to allow for microstructures, and a quasi-convexification of the integrand in $\mathcal{J}[u, \mathcal{O}]$ (by taking the infimum over all possible microstructures) then ensures existence of minimizers [3].

There are various approximations and implementations of the elastic shape optimization problem, each of which more or less corresponds to a particular type of regularization. A direct triangulation of $\mathcal{O}$ or its boundary would probably work with all regularizations, but would require remeshing during the optimization and induce technical difficulties with topological changes. The so-called evolutionary structural optimization (ESO) is based on discretizing the computational domain by finite elements and successively removing those elements which contribute least to the structural stiffness (or another chosen objective, see for example [10]). This corresponds to a regularization via discretization and thereby introduces a mesh dependence. 
The implicit representation of shapes via level sets and corresponding shape optimization approaches are investigated by various authors [4,23,25,29]. In particular Allaire and coworkers $[1,3,4,7]$ studied level-set methods in two- and three-dimensional structural optimization and combined this approach with a homogenization method. In [5] they also investigated topological optimization in the context of minimizing the expected elastic stress. Shape sensitivity analysis as introduced by Sokolowski and Zolésio [31] can be phrased elegantly in terms of level sets. For the relaxation of the shape functional $\mathcal{O} \mapsto \mathcal{J}[u[\mathcal{O}], \mathcal{O}]$ gradient-descent schemes have been investigated, where the actual shape gradient and thus the performance of the relaxation scheme significantly depend on the underlying metric $g$. Burger and Stainko [15] provide examples for different $g$ such as the inner product in $H^{1}(\partial \mathcal{O})$ (Laplace-Beltrami flow), $H^{1 / 2}(\partial \mathcal{O})$ (Stefan flow), $L^{2}(\partial \mathcal{O})$ (Hadamard flow), $H^{-1 / 2}(\partial \mathcal{O}$ ) (Mullins-Sekerka flow), and $H^{-1}(\partial \mathcal{O})$ (surface diffusion flow). Allaire et al. [6] also propose to use $H^{1}-$, $L^{2}-$, or $H^{-1}$-type inner products. Indeed, during the gradient flow, topology changes can only happen by merging or eliminating holes (whereas in $3 \mathrm{D}$, holes may appear by pinching a thin wall) $[2,6]$ so that the maximum number of holes is prescribed by the initialization. In order to allow the level-set method to create holes in $2 \mathrm{D}$, the topological derivative is sometimes used to identify and remove rather inactive interior material parts[7,20,24].

An implicit description of shapes via phase fields - the approach also employed in this paper - is both analytically and numerically an attractive alternative. Phase-field models originated in the physical description of multiphase materials: The chemical bulk energy of the material is given by $\frac{1}{\varepsilon} \int_{\Omega} \Psi(v) \mathrm{d} x$ for some chemical potential $\Psi$. The minima of $\Psi$ represent two material phases. This energy is complemented by an interfacial energy of the form $\varepsilon \int_{\Omega}|\nabla v|^{2} \mathrm{~d} x$. Optimal profiles of $v$ show a diffusive transition region between the two material phases whose width scales with the parameter $\varepsilon$. For $\varepsilon \rightarrow 0$, the above integral forces the phase field $v$ towards the pure phases, and for appropriate choices of $\Psi$ it $\Gamma$-converges to the total interface length. Hence, the approach lends itselfto a perimeter regularization. This technique is employed by Wang and Zhou [32] who minimize the compliance of an elastic structure using a triphasic phase field (with one void and two material phases) for which the potential $\Psi$ is equipped with a periodically repeated sequence of three minima to allow for all three possible types of phase transitions. Furthermore, they replace the term $\int_{\Omega}|\nabla v|^{2} \mathrm{~d} x$ by an edgepreserving smoothing and perform a multiscale relaxation, starting with large $\varepsilon$ and successively decreasing it - remarkably beyond the point up to which the grid can still resolve the diffusive interface in a usual fashion. Zhou and Wang [37] compute the Cahn-Hilliard evolution of the shape to be optimized, also using a multiphase material. They solve the elastic equations with finite elements and the resulting fourth-order CahnHilliard-type partial differential equation with a Crank-Nicolson finite-difference scheme in which nonlinear terms are approximated by Taylor series expansion and the resulting linear system is solved by a multigrid V-cycle. Burger and Stainko [15] minimize the volume $|\mathcal{O}|$ under a stress constraint and show existence of a corresponding minimizer. They use a double obstacle potential $\Psi$ to reformulate the shape optimization as a quadratic programming problem with linear constraints. Finally, Bourdin and Chambolle [13] find minimum compliance designs for (design-dependent) pressure loads, using a solid, liquid, and void phase, which they describe by a scalar phase field allowing for the transitions void-solid-liquid. They also prove existence of minimizers for the sharp-interface model and implement the optimization as a semi-implicit descent scheme with linear finite elements on a triangular unstructured mesh.

Guo et al. [24] describe the characteristic function of $\mathcal{O}$ by the concatenation of a smoothed Heaviside function with a level-set function, where the smoothed Heaviside function acts like a phase-field profile. Wei and Wang [35] encode $\mathcal{O}$ in a piecewise constant level set function $v$, which is also closely related to the phasefield method: They regularize $v$ via total variation, which in conjunction with the penalty $\int_{\Omega}(v-1)^{2}(v-2)^{2} \mathrm{~d} x$ for the constraint $v \in\{1,2\}$ has a similar effect as the phase-field perimeter term $\frac{1}{2} \int_{\Omega} \varepsilon|\nabla v|^{2}+\frac{1}{\varepsilon} \Psi(v) \mathrm{d} x$. Xia and Wang [36] compute functionally graded structures, where the shape is described by a level set function and the smoothly varying material properties by a scalar field (that actually describes the mixture of two material components from which the physical properties are computed under an isotropy assumption). 


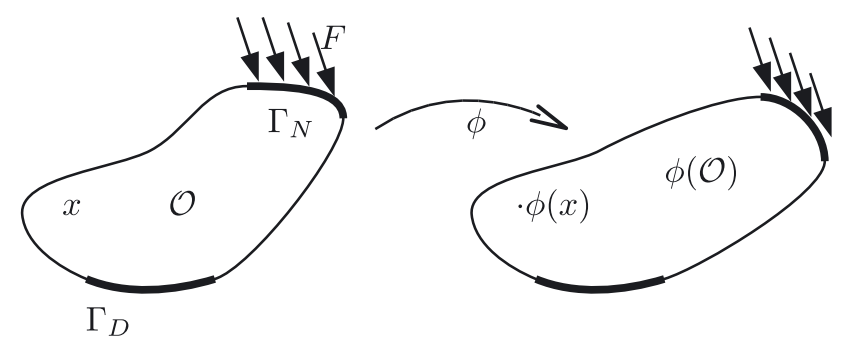

Figure 1. A surface load $F$ induces a deformation $\phi$ of the body $\mathcal{O}$.

\section{The NONLINEAR ELASTIC SHAPE OPTIMIZATION MODEL}

In this section, we will briefly recapitulate the mechanical framework of nonlinear elasticity and discuss the different associated compliance cost functionals.

\subsection{The hyperelastic constitutive law}

Let us assume we are given a sufficiently regular, elastic body $\mathcal{O} \subset \mathbb{R}^{d}$ (in the following, we will only consider $d=3$, the transfer to $d \neq 3$ being obvious) which is fixed at part of its boundary, $\Gamma_{D} \subset \partial \mathcal{O}$, and subjected to a sufficiently regular surface load $F: \Gamma_{N} \rightarrow \mathbb{R}^{d}$ on $\Gamma_{N} \subset \partial \mathcal{O}$ (Fig. 1). Throughout the paper we will only consider dead loads that do not change their intensity or direction during a deformation. The body deforms under the surface load, and the equilibrium deformation $\phi: \mathcal{O} \rightarrow \mathbb{R}^{d}$ minimizes the total free energy

$$
\mathcal{E}[\mathcal{O}, \phi]=\mathcal{W}[\mathcal{O}, \phi]-\mathcal{C}[\phi]
$$

within a set of admissible deformations $\phi$ with trace $\left.\phi\right|_{\Gamma_{D}}=\mathrm{id}$, where

$$
\mathcal{W}[\mathcal{O}, \phi]=\int_{\mathcal{O}} W(\mathcal{D} \phi) \mathrm{d} x
$$

describes the elastic energy stored inside the material and

$$
\mathcal{C}[\phi]=\int_{\Gamma_{N}} F \cdot(\phi-\mathrm{id}) \mathrm{d} a
$$

is the (negative) potential of the surface load. Here, we postulated the existence of a Gibbs free energy density which only depends on the Jacobian $\mathcal{D} \phi$ of the deformation, also denoted the deformation gradient. Materials for which this assumption holds are called hyperelastic.From the frame-indifference principle and the assumption of an isotropic material one deduces that the energy density $W$ only depends on the singular values, the socalled principal stretches, $\lambda_{1}, \lambda_{2}, \lambda_{3}$ of the deformation gradient $\mathcal{D} \phi$. Instead of the principal stretches, we can equivalently describe the local deformation using the so-called invariants of the deformation gradient or the Cauchy-Green strain tensor $\mathcal{D} \phi^{T} \mathcal{D} \phi$,

$$
I_{1}=\|\mathcal{D} \phi\|_{F}=\sqrt{\lambda_{1}^{2}+\lambda_{2}^{2}+\lambda_{3}^{2}}, \quad I_{2}=\|\operatorname{cof} \mathcal{D} \phi\|_{F}=\sqrt{\lambda_{1}^{2} \lambda_{2}^{2}+\lambda_{1}^{2} \lambda_{3}^{2}+\lambda_{2}^{2} \lambda_{3}^{2}}, \quad I_{3}=\operatorname{det} \mathcal{D} \phi=\lambda_{1} \lambda_{2} \lambda_{3},
$$

where $\|A\|_{F}=\sqrt{\operatorname{tr}\left(A^{\mathrm{T}} A\right)}$ for $A \in \mathbb{R}^{d \times d}$ and the cofactor matrix is given by $\operatorname{cof} A=\operatorname{det} A A^{-\mathrm{T}}$ for $A \in G L(d)$, so that overall, for an appropriately chosen $\hat{W}$ we obtain

$$
W(\mathcal{D} \phi)=\hat{W}\left(I_{1}, I_{2}, I_{3}\right)
$$


$I_{1}, I_{2}$, and $I_{3}$ can be interpreted as the locally averaged change of an infinitesimal length, area, and volume during the deformation, respectively.

The elastic energy densities have to fulfill further conditions. First, we require the identity, $\mathcal{D} \phi=\mathbb{I}$ (which corresponds to no displacement), to be the global minimizer. Second, the energy density shall converge to infinity as $I_{3}$, the determinant of the deformation gradient (which describes the volume change), approaches zero or infinity. Negative values of $I_{3}$ correspond to local interpenetration of matter and are not allowed at all. Thus, $W(\mathcal{D} \phi)=\hat{W}\left(I_{1}, I_{2}, I_{3}\right)$ is strongly nonlinear and can in addition not be convex in the deformation gradient $\mathcal{D} \phi$, since the set of matrices with positive determinant is not even a convex set [18]. This makes the problem of existence of minimizers a subtle one, but it can be treated using the direct method of the calculus of variations [11]: Assuming $W(\mathcal{D} \phi) \geq C_{1}\|\mathcal{D} \phi\|_{F}^{p}-C_{2}$ for some $p>1, C_{1}, C_{2}>0$, as well as $F \in L^{p^{\prime}}\left(\Gamma_{N}\right)$ for $1=\frac{1}{p}+\frac{1}{p^{\prime}}$, and using the boundedness of the trace operator $W^{1, p}(\mathcal{O}) \rightarrow L^{p}\left(\Gamma_{N}\right)$, we directly verify the weak coercivity of $\mathcal{E}[\mathcal{O}, \cdot]$ on $W^{1, p}(\mathcal{O})$. For the weak lower semi-continuity of $\mathcal{E}[\mathcal{O}, \cdot]$ we have to require lower semi-continuity of $\mathcal{W}$, which translates into quasiconvexity of $W$ [21]. We will here employ a slightly stronger requirement [11], the polyconvexity of $W$ (that is, $W(\mathcal{D} \phi)$ can be rewritten as a convex function of all minors of the deformation gradient $\mathcal{D} \phi)$ so that $\mathcal{W}[\mathcal{O}, \cdot]$ is weakly lower semi-continuous on $W^{1, p}(\mathcal{O})$ for $p \geq d[14]$, and the variational problem $\min _{\phi} \mathcal{E}[\mathcal{O}, \phi]$ indeed admits a minimizer in $\left\{\phi \in W^{1, p}(\mathcal{O}):\left.\phi\right|_{\Gamma_{D}}=\right.$ id $\}$. By imposing growth conditions of the form $W(A) \geq C_{1}\left(\|A\|_{F}^{p}+\|\operatorname{cof} A\|_{F}^{q}+|\operatorname{det} A|^{r}\right)-C_{2}$ one can obtain existence results for smaller $p$ under appropriate conditions on $q$ and $r$ [28]. Typical energy densities of the above type are given by

$$
W(\mathcal{D} \phi)=\hat{W}\left(I_{1}, I_{2}, I_{3}\right)=a_{1}\|\mathcal{D} \phi\|_{F}^{p}+a_{2}\|\operatorname{cof} \mathcal{D} \phi\|_{F}^{q}+Z(\operatorname{det} \mathcal{D} \phi)
$$

for $a_{1}, a_{2}>0, p, q>1$, and a convex function $Z: \mathbb{R} \rightarrow \mathbb{R}$ with $\lim _{s \rightarrow \infty} Z(s)=\lim _{s \rightarrow 0} Z(s)=\infty$. For example, $p=2$ and $q=0$ yields a neo-Hookean material law, while $p=q=2$ results in a Mooney-Rivlin material law [18]. In our computations, we will employ the particular material law $W(\mathcal{D} \phi)=\frac{\mu}{2}\|\mathcal{D} \phi\|_{F}^{2}+\frac{\lambda}{4} \operatorname{det} \mathcal{D} \phi^{2}-$ $\left(\mu+\frac{\lambda}{2}\right) \log \operatorname{det} \mathcal{D} \phi-\frac{d \mu}{2}-\frac{\lambda}{4}$ for material parameters $\lambda$ and $\mu$, whose second order Taylor expansion about $\mathcal{D} \phi=\mathbb{I}$ (which reveals the small-strain behavior) yields the standard energy from isotropic linearized elasticity, $W^{\text {lin }}(\mathcal{D} \phi)=\frac{\lambda}{2}(\operatorname{tr} \epsilon)^{2}+\mu \operatorname{tr}(\epsilon)^{2}$, with $\epsilon=\frac{1}{2}\left((\mathcal{D} \phi-\mathbb{I})+(\mathcal{D} \phi-\mathbb{I})^{\mathrm{T}}\right)$ and the Lamé constants $\lambda$ and $\mu$.

In general linearized elasticity the energy density of the material is a quadratic function

$$
W^{\operatorname{lin}}(\mathcal{D} \phi)=\frac{1}{2} \mathbf{C}(\mathcal{D} \phi-\mathbb{I}):(\mathcal{D} \phi-\mathbb{I})
$$

of thedisplacement gradient $(\mathcal{D} \phi-\mathbb{I})$, where $\mathbf{C}$ denotes a fourth order symmetric positive (semi-)definite (elasticity) tensor and $A: B=\operatorname{tr} A^{T} B$. Hence, we obtain the equilibrium condition $0=\int_{\mathcal{O}} \mathbf{C}(\mathcal{D} \phi-\mathbb{I})$ : $\mathcal{D} \theta \mathrm{d} x-\int_{\Gamma_{N}} F \cdot \theta \mathrm{d} a$ for all test displacements $\theta$. In particular, this holds for $\theta=\phi-$ id, which implies $2 \mathcal{W}^{\operatorname{lin}}[\mathcal{O}, \phi]=\mathcal{C}[\phi]$ for the equilibrium deformation $\phi$. Here, $2 \mathcal{W}^{\operatorname{lin}}[\mathcal{O}, \phi]=\mathcal{C}[\phi]$ represents a measure of the deformation strength and is denoted the compliance of the object $\mathcal{O}$, which may be seen as some kind of inverse rigidity. As discussed in Section 2 it is mostly this compliance which has been minimized in elastic shape optimization. Obviously, in linearized elasticity, it does not matter whether we describe the compliance via $2 \mathcal{W}^{\operatorname{lin}}[\mathcal{O}, \phi]$ or $\mathcal{C}[\phi]$. However, in the case of a nonlinear hyperelastic constitutive law, this is no longer true as we will see later and it makes a difference which term we choose to minimize.

\subsection{The shape optimization problem based on compliance minimization}

If we ask for elastic domains $\mathcal{O} \subset \Omega \subset \mathbb{R}^{d}$ which minimize the compliance, then, certainly, $\mathcal{O} \equiv \Omega$ yields the most rigid structure. Hence, we are actually interested in a balance between rigidity, material consumption (weight), and manufacturing simplicity (smoothness of the shape $\partial \mathcal{O}$ ). The material consumption is expressed by the Lebesgue measure of $\mathcal{O}, \mathcal{V}[\mathcal{O}]=|\mathcal{O}|$. Domains $\mathcal{O}$ that minimize just a weighted sum of compliance and volume in general do not exist. Typically, microstructures appear (cf. Sect. 2), for example higher rank sequential laminates in which material and void rapidly alternate. Hence, as already discussed above, we replace the void and adjust the elastic energy $\mathcal{W}[\mathcal{O}, \phi]$ accordingly by defining $\mathcal{W}^{\delta}[\mathcal{O}, \phi]=\int_{\Omega}\left((1-\delta) \chi_{\mathcal{O}}+\delta\right) W(\mathcal{D} \phi) \mathrm{d} x$. 
Furthermore, we add the domain perimeter $\mathcal{L}[\partial \mathcal{O}]=\mathcal{H}^{d-1}(\partial \mathcal{O})$ as a regularizing prior, which can be interpreted as introducing manufacturing costs for the production and processing of the object surface. Now, the total free energy is given by

$$
\mathcal{E}^{\delta}[\mathcal{O}, \phi]=\mathcal{W}^{\delta}[\mathcal{O}, \phi]-\mathcal{C}[\phi]
$$

In our computations we choose $\delta=10^{-4}$. Let us remark that this modification of the free energy in particular allows to properly define the deformation $\phi$ outside $\mathcal{O}$ and (combined with suitable Dirichlet boundary conditions on $\partial \Omega$ ) to prevent self-penetration of matter in form of overlapping material parts. In the case of compliance minimization via the homogenization method, the shape optimization with real void has been examined as the limit case when the stiffness of the weak material tends to zero [3].

As already explained earlier, the compliance of an object $\mathcal{O}$ may be regarded as a kind of inverse rigidity and can in linearized elasticity be described as $2 \mathcal{W}^{\operatorname{lin}}[\mathcal{O}, \phi]$ or equivalently $\mathcal{C}[\phi]$ for the equilibrium deformation $\phi$. In nonlinear elasticity, however, $\mathcal{W}[\mathcal{O}, \phi]$ and $\mathcal{C}[\phi]$ are no longer related by a factor of 2 , and the question arises which one appropriately corresponds to the compliance in the linearized setting and which one should be chosen for shape optimization. The stored elastic energy $\mathcal{W}[\mathcal{O}, \phi]$ corresponds to the work transferred to the body $\mathcal{O}$ by the surface load, while the total decrease $\mathcal{C}[\phi]$ in the potential energy of the surface load $F$ is composed of exactly this work plus the energy dissipation during the system dynamics before the equilibrium is reached. Allaire etal. [6], who have already computed a nonlinearly elastic shape optimization example, consider the minimization of the surface load potential $\mathcal{C}[\phi]$. Here, we aim at an analysis of the differences and consider $($ for parameters $\eta, \nu>0$ ) both cases, the minimization of the total potential energy of the surface load

$$
\mathcal{J}_{\mathcal{C}}[\mathcal{O}, \phi]:=\mathcal{C}[\phi]+\nu \mathcal{V}[\mathcal{O}]+\eta \mathcal{L}[\partial \mathcal{O}]
$$

or the stored elastic deformation energy

$$
\mathcal{J}_{\mathcal{W}}[\mathcal{O}, \phi]:=2 \mathcal{W}^{\delta}[\mathcal{O}, \phi]+\nu \mathcal{V}[\mathcal{O}]+\eta \mathcal{L}[\partial \mathcal{O}]
$$

However, there is a third possibility which also reduces to the standard notion of compliance in linearized elasticity. Indeed, we might also choose to minimize the dissipation associated with the transition from the unstressed state to the equilibrium deformation, $-2 \mathcal{E}^{\delta}=2\left(\mathcal{C}-\mathcal{W}^{\delta}\right)$. Together with the volume and surface regularization we obtain

$$
\mathcal{J}_{\mathcal{D}}[\mathcal{O}, \phi]:=2 \mathcal{C}[\phi]-2 \mathcal{W}^{\delta}[\mathcal{O}, \phi]+\nu \mathcal{V}[\mathcal{O}]+\eta \mathcal{L}[\partial \mathcal{O}]
$$

Independent of the specific cost functional we always consider a minimization for $\mathcal{O} \in\left\{\mathcal{O} \subset \Omega: \Gamma_{D}, \Gamma_{N} \subset \partial \mathcal{O}\right\}$ under the constraint that $\phi: \Omega \rightarrow \mathbb{R}^{d}$ minimizes the free energy $\mathcal{E}^{\delta}[\mathcal{O}, \phi]$ among all deformations in the associated admissible set of deformations whose trace is the identity on $\Gamma_{D}$.

The following toy problem shall illustrate the conceptual differences at least of the first two cases: Consider the task to design a structure $\mathcal{O}$ which is attached to a wall at its left end and has to bear a vertical load $F$ at its right end (Fig. 2). A cantilever-like design (Fig. 2, left half) exhibits a rather small displacement $\phi-$ id and thus a small value of $\mathcal{C}[\phi]$, but the strong compression of the lower branch causes a relatively high deformation energy $\mathcal{W}[\mathcal{O}, \phi]$. A freely rotating rod, on the other hand, allows a strong displacement with high $\mathcal{C}[\phi]$ but low $\mathcal{W}[\mathcal{O}, \phi]$ (Fig. 2, right half). The former design is more appropriate if the load is supposed to be sustained without large displacements while the latter design is more related to the material strain and is useful in systems where the energy dissipation on the way to the final equilibrium configuration is absorbed by a reasonable damping mechanism. With respect to the applications considered in this paper, we have to keep in mind that shape optimization with respect to $\mathcal{C}[\phi]$ will yield results of the same type as in Figure 2, left, while optimization with respect to $\mathcal{W}[\mathcal{O}, \phi]$ generally allows strong deformations and tends to produce shapes as in Figure 2, right. Let us remark, however, that minimizing $\mathcal{W}[\mathcal{O}, \phi]$ does not always result in designs with strong displacements. For instance in case of sufficiently low volume costs, $\mathcal{O} \equiv \Omega$ will yield the optimal design. 


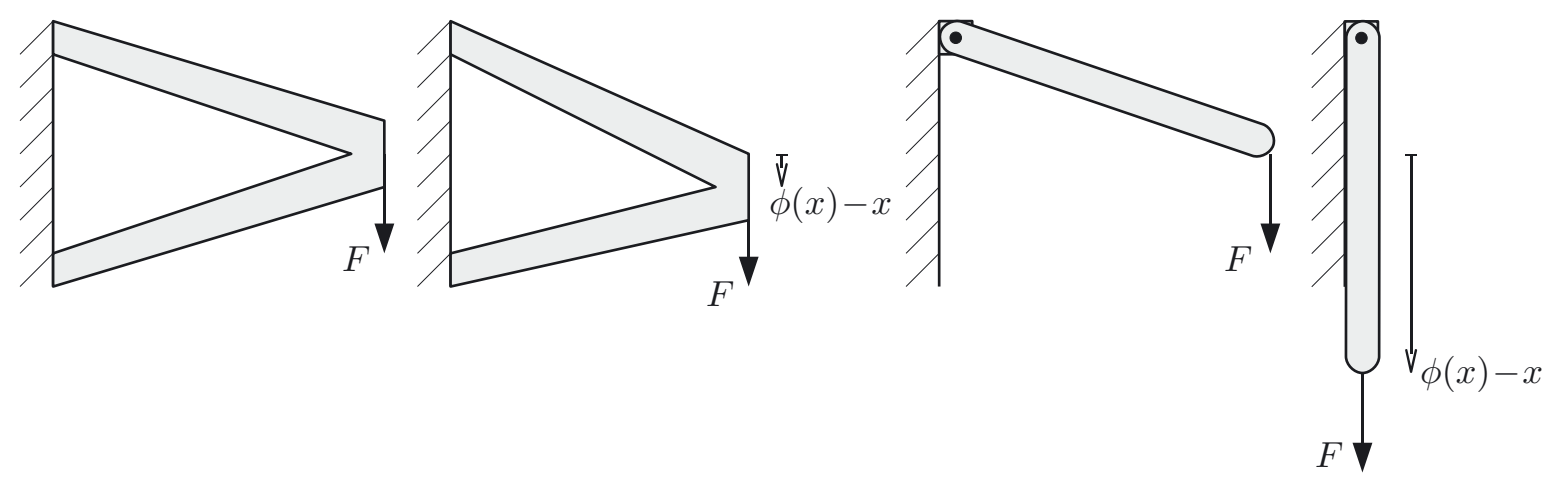

Figure 2. Two possible designs (each shown in the undeformed and the deformed state) to bear a vertical load. The left design exhibits rather low $\mathcal{C}[\phi]$, but high $\mathcal{W}[\mathcal{O}, \phi]$ whereas the right design (with a hinge) yields the reverse.

\subsection{The approximating phase-field model for shape optimization}

Shape and topology optimization based on an explicit parametric description of the mechanical devices is algorithmically very difficult, as already mentioned above. Hence, we consider an approximating phase-field representation of the object $\mathcal{O}$ via a phase-field function $v: \Omega \rightarrow \mathbb{R}$ of Allen-Cahn or Modica-Mortola type. Such phase fields constitute a convenient implicit representation of the objects and their complement and allow for a simple approximation of their boundary length. They originate from the description of biphasic materials with a diffusive interface layer, where $v$ might for example represent the local concentration of a chemical constituent. The local chemical energy density $\Psi(\cdot)$ has two minima corresponding to the two pure phases. The resulting total bulk energy $\int_{\Omega} \Psi(v) \mathrm{d} x$ is then perturbed by an additional interfacial energy of the form $\int_{\Omega}|\nabla v|^{2} \mathrm{~d} x$. In our context of shape modeling, we assume that the two minima of $\Psi$ are -1 and 1 with $\Psi(-1)=\Psi(1)=0$ representing the two phases void (outside of $\mathcal{O}$ ) and material (inside of $\mathcal{O}$ ), respectively. Using a proper scaling we obtain the free phase-field energy

$$
\mathcal{L}_{\mathrm{MM}}^{\varepsilon}[v]=\frac{1}{2} \int_{\Omega} \varepsilon|\nabla v|^{2}+\frac{1}{\varepsilon} \Psi(v) \mathrm{d} x
$$

where the scale parameter $\varepsilon$ describes the width of the interfacial region. In the limit $\varepsilon \rightarrow 0$, the phase field $v$ is forced towards the pure phases -1 and 1 and $\Gamma$-converges to a multiple of the total interface area. In fact, for $\Psi \in C^{1}(\mathbb{R})$ with $\Psi(-1)=\Psi(1)=0$ being the global minima, the following rigorous result holds [14]: If $\mathcal{L}_{\mathrm{MM}}^{\varepsilon}[v]:=\infty$ for $v \notin W^{1,2}(\Omega)$, then

$$
\Gamma-\lim _{\varepsilon \rightarrow 0} \mathcal{L}_{\mathrm{MM}}^{\varepsilon}[\cdot]=c_{\Psi} \operatorname{Per}(\cdot), \quad c_{\Psi}=\int_{-1}^{1} \sqrt{\Psi(s)} \mathrm{d} s,
$$

with respect to the $L^{1}(\Omega)$-topology and for $\operatorname{Per}(w):=\mathcal{H}^{d-1}(\Omega \cap \partial\{x \in \Omega: w(x)=1\})$ if $w: \Omega \rightarrow\{-1,1\}$ almost everywhere and $\operatorname{Per}(w):=\infty$ else.Here, $\partial$ denotes the measure-theoretic essential boundary. In (3.1), (3.2), and (3.3) the perimeter term $\mathcal{L}[\partial \mathcal{O}]$ is then replaced by $\mathcal{L}_{\mathrm{MM}}^{\varepsilon}[v]$, where we choose the double-well potential

$$
\Psi(v)=\frac{9}{16}\left(v^{2}-1\right)^{2},
$$

which yields $c_{\Psi}=1$. Furthermore, we introduce an approximation $\chi_{\mathcal{O}}(v)$ to the characteristic function $\chi_{\mathcal{O}}$, choosing

$$
\chi_{\mathcal{O}}(v)=\frac{1}{4}(v+1)^{2} .
$$


With this function at hand we approximate the total volume by $\mathcal{V}[v]=\int_{\Omega} \chi_{\mathcal{O}}(v) \mathrm{d} x$ and the stored elastic energy by $\mathcal{W}^{\delta}[v, \phi]:=\int_{\Omega}\left((1-\delta) \chi_{\mathcal{O}}(v)+\delta\right) W(\mathcal{D} \phi) \mathrm{d} x$, where we use with a slight misuse of notation the same symbol for the energy terms in the phase-field model as in the original problem. Overall, based on the phase-field approximation we will now minimize one of the following three functionals

$$
\begin{aligned}
\mathcal{J}_{\mathcal{W}}^{\varepsilon}[v, \phi] & =2 \mathcal{W}^{\delta}[v, \phi]+\nu \mathcal{V}[v]+\eta \mathcal{L}_{\mathrm{MM}}^{\varepsilon}[v], \\
\mathcal{J}_{\mathcal{C}}^{\varepsilon}[v, \phi] & =\mathcal{C}[\phi]+\nu \mathcal{V}[v]+\eta \mathcal{L}_{\mathrm{MM}}^{\varepsilon}[v], \\
\mathcal{J}_{\mathcal{D}}^{\varepsilon}[v, \phi] & =2\left(\mathcal{C}[\phi]-\mathcal{W}^{\delta}[v, \phi]\right)+\nu \mathcal{V}[v]+\eta \mathcal{L}_{\mathrm{MM}}^{\varepsilon}[v],
\end{aligned}
$$

for integrable functions $v: \Omega \rightarrow \mathbb{R}$ with $\left.v\right|_{\Gamma_{D} \cup \Gamma_{N}}=1$ under the constraint that $\phi: \Omega \rightarrow \mathbb{R}^{d}$ with $\left.\phi\right|_{\Gamma_{D}}=$ id minimizes the total free energy for a phase field $v$, defined (again with a slight misuse of notation) as

$$
\mathcal{E}^{\delta}[v, \phi]:=\mathcal{W}^{\delta}[v, \phi]-\mathcal{C}[\phi] .
$$

\section{EFFECTS OF NONLINEAR ELASTICITY AND THEIR IMPACT ON THE SHAPE OPTIMIZATION}

The use of a nonlinear instead of a linearized elasticity changes the nature of the compliance minimization problem qualitatively. In the following, we will investigate the symmetry-breaking effect of nonlinear elasticity on the optimal shapes and the presence of buckling instabilities which are typical for the nonlinear models. This discussion is meant as a motivation for the analytical treatment in Section 5 and underpins the specific design of the numerical algorithm presented in Section 6.

\subsection{Break of symmetry in nonlinear elastic shape optimization}

As a first feature that distinguishes nonlinear from linearized elasticity, let us consider the effect of a sign change of the load $F$ and its nonlinear impact not only on the deformation but also on the optimal geometry $\mathcal{O}$. In linearized elasticity, the (unique) equilibrium deformation is the minimizer of the free energy

$$
\mathcal{E}_{F}^{\operatorname{lin}}[\mathcal{O}, \phi]=\mathcal{W}^{\operatorname{lin}}[\mathcal{O}, \phi]-\mathcal{C}_{F}[\phi]:=\int_{\mathcal{O}} \frac{1}{2} \mathbf{C}(\mathcal{D} \phi-\mathbb{I}):(\mathcal{D} \phi-\mathbb{I}) \mathrm{d} x-\int_{\Gamma_{N}} F \cdot(\phi-\mathrm{id}) \mathrm{d} a
$$

for the symmetric, positive semi-definite elasticity tensor $\mathbf{C}$, where the subscript $F$ indicates the surface load. Obviously, if $\phi_{F}$ minimizes $\mathcal{E}_{F}^{\text {lin }}[\mathcal{O}, \cdot]$, then $\phi_{-F}:=2$ id $-\phi_{F}$ minimizes $\mathcal{E}_{-F}^{\text {lin }}[\mathcal{O}, \cdot]$, the total free energy for a reversed direction of the surface load. Indeed, for deformations $\phi, \psi$ with $\phi+\psi=2$ id we have $\mathcal{E}_{F}^{\operatorname{lin}}[\mathcal{O}, \phi]=$ $\mathcal{E}_{-F}^{\operatorname{lin}}[\mathcal{O}, \psi], \mathcal{W}^{\operatorname{lin}}[\mathcal{O}, \phi]=\mathcal{W}^{\operatorname{lin}}[\mathcal{O}, \psi]$, and $\mathcal{C}_{F}[\phi]=\mathcal{C}_{-F}[\psi]$. Hence, the optimal geometry $\mathcal{O}$ for a prescribed load $F$ is the same one as for the load $-F$. As a consequence, if the sign change of $F$ has the same effect as mirroring the shape optimization problem (for example, for the cantilever design problem in Fig. 3), then the resulting optimal shapes are symmetric. In contrast, in nonlinear elasticity, the material behavior and geometry change depend strongly on whether we tear at or push against an object. A sign change of the load $F$ no longer simply implies a sign change of the displacement. Consequently, the symmetry property of linearized elasticity is lost: Where the shape optimization with linearized elasticity results in a symmetric shape $\mathcal{O}$, the corresponding optimal geometry for nonlinear elasticity is generally asymmetric. This phenomenon can already be observed for quite small displacements as shown in Figure 3 for the design of a cantilever, where the effect of gradually increasing the load $F$ is explored.

Note that in this example the lower, compressed rods are thinner than the upper, dilated structures, which might at first sight seem counter-intuitive. However, consider the simple toy problem from Figure 4, where a load $F$ is suspended from a cantilever consisting of two rods with length $l$ and a square cross-section of width $d_{1}$ and $d_{2}$, respectively, that are connected to each other and to the wall by a joint. Let us assume the rods to be linearly elastic with elastic modulus $E$ and the force $F$ to be small enough so that the lower rod does not buckle out (compare the paragraph below as well as the next section). If we denote in a 3D configuration 

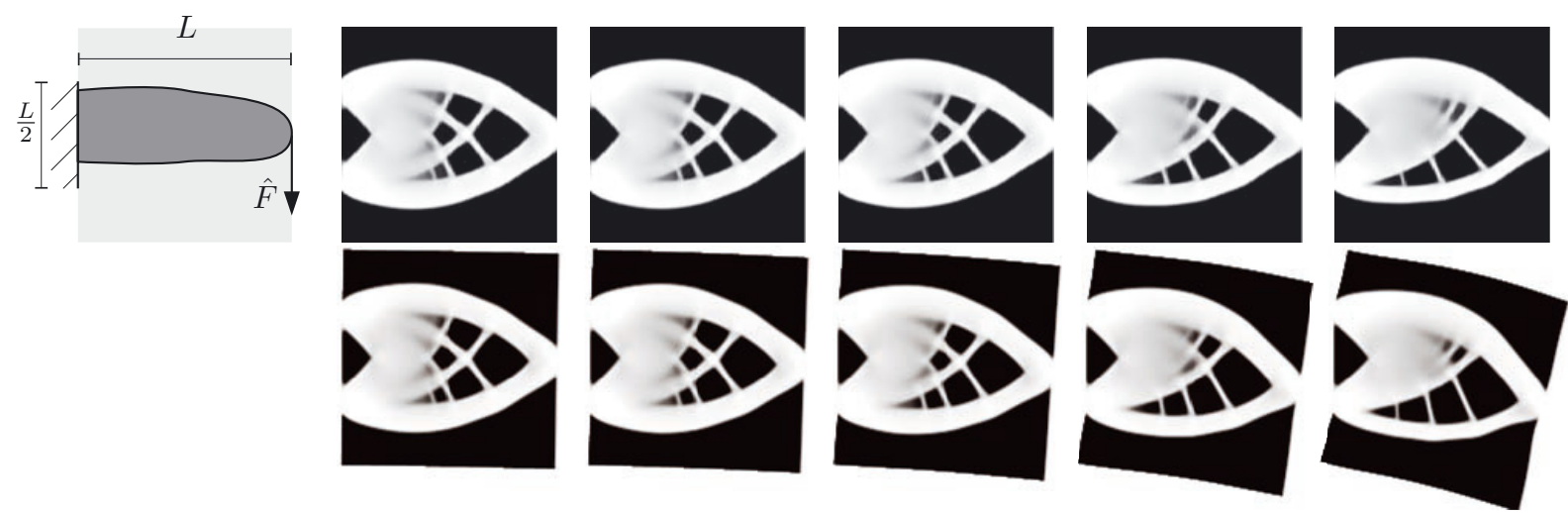

Figure 3. Optimal design of a cantilever according to the sketch top left. The top row shows optimal designs for loads $\hat{F}=0.5,1,2,4,6$ (the point load $\hat{F}$ is approximated by a tent-like surface load $F$ along a width of $2^{-3}$ ) and $\eta=2^{5} \cdot 10^{-5} \cdot \hat{F}^{2}, \nu=2^{10} \cdot 10^{-4} \cdot \hat{F}^{2}$ (underlying grid resolution $257^{2}, \lambda=\mu=80, L=1$ ). The bottom row shows the equilibrium deformation. In linearized elasticity, all parameter combinations would yield exactly the same symmetric, optimal shape whereas here, we see strong asymmetries evolving. Computations were performed using the phase-field model. White indicates full material while black represents the weak material whose stiffness is reduced by the factor $\delta=10^{-4}$. Note: In the region at the left wall where Dirichlet boundary conditions for the deformation are applied, the algorithm realizes that stiff material in the center of this region does not contribute much to the structural rigidity and hence removes it.

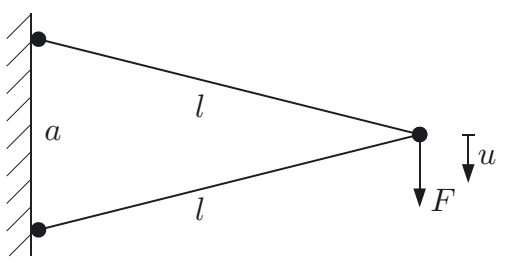

FiguRE 4. Simple model of a cantilever with joints at the rod ends. The structure width is $2 a$ so that $l=\frac{\sqrt{17}}{2} a$.

the rod elongation by $\lambda_{1}$ and $\lambda_{2}$ and the load displacement by $u$, then the total free energy is given by

$$
\mathcal{E}\left[\lambda_{1}, \lambda_{2}\right]=\frac{1}{2} \operatorname{Eld}_{1}^{2}\left(\lambda_{1}-1\right)^{2}+\frac{1}{2} \operatorname{Eld}_{2}^{2}\left(\lambda_{2}-1\right)^{2}-F u=\frac{1}{2} \operatorname{Eld}_{1}^{2}\left(\lambda_{1}-1\right)^{2}+\frac{1}{2} \operatorname{Eld}_{2}^{2}\left(\lambda_{2}-1\right)^{2}-\frac{17}{8} a F\left(\lambda_{1}^{2}-\lambda_{2}^{2}\right),
$$

and its minimization yields the stretches $\lambda_{1 / 2}=\left(1 \mp \frac{\sqrt{17}}{2} \frac{F}{E d_{1 / 2}^{2}}\right)^{-1}$. Now let the structure volume be fixed, $V=l\left(d_{1}^{2}+d_{2}^{2}\right)$ or equivalently $d_{2}=\left(\frac{V}{l}-d_{1}^{2}\right)^{\frac{1}{2}}$, and consider as compliance objective functional the work $\mathcal{J}_{\mathcal{C}}\left[d_{1}\right]=F u=\frac{17}{8} a F\left(\lambda_{1}^{2}-\lambda_{2}^{2}\right)$. The minimizing $d_{1}$ is always larger than the corresponding $d_{2}=\left(\frac{V}{l}-d_{1}^{2}\right)^{\frac{1}{2}}$, which can for example be seen by transforming the optimality condition $0=\delta_{d_{1}} \mathcal{J}_{\mathcal{C}}\left[d_{1}\right]$ into the equation $\frac{d_{1}^{2}}{\left(d_{1}^{2}-\frac{\sqrt{17}}{2} \frac{F}{E}\right)^{3}}=\frac{d_{2}^{2}}{\left(d_{2}^{2}+\frac{\sqrt{17}}{2} \frac{F}{E}\right)^{3}}$. Hence, for (comparatively) small forces, the lower rod will be thinner than the upper rod, in accordance with our experiments.

For stronger forces, the lower rod will buckle out if it is too thin, which results in a strong increase of the objective energy $\mathcal{J}_{\mathcal{C}}$. Hence, in the case of stronger forces, the optimal thickness of the lower rod will be just below the buckling thickness, and the upper rod hence ends up to be thinner than the bottom one due 
to the volume constraint, which represents the perhaps more intuitive case that is apparently also obtained in [6]. The above toy problem can be adapted to a simple approximate geometrically nonlinear model and then will indeed reflect this buckling phenomenon as well. For even stronger forces, where the bottom rod buckles out anyway, it would again be optimal to make the upper rod thicker.

\subsection{Buckling instabilities}

A further, even more striking phenomenon of nonlinear elasticity is associated with the non-uniqueness of equilibrium deformations: While in linearized elasticity the total free energy $\mathcal{E}[\mathcal{O}, \phi]$ is convex and quadratic in the deformation $\phi$, the energy landscape is much more complicated in nonlinear elasticity and generally admits multiple (locally) minimizing deformations $\phi$. Of course, this raises the question which one of the equilibrium deformations we should actually consider during shape optimization, and this will be investigated theoretically in Section 5.2. Often, the existence of multiple, locally minimizing deformations comes along with the bending of structures. The classical example is given by the compression of straight beams [26], which we recapitulate here to prepare a later discussion of the non-existence of optimal shapes in a worst-case scenario.

Consider a straight bar of length $L$ which is clamped at one end and subjected to a compression load $F$ at the other end. Let us denote the displacement orthogonal to the bar at position $x \in[0, L]$ by $u(x)$, then the bending moment $M(x)$ inside the bar at $x$ is given by $M(x)=F(u(L)-u(x))$. Under the assumption of Bernoulli's beam hypothesis and Hooke's law with Young's modulus $E$, this moment can also be expressed as $M(x)=\frac{E I}{\rho}$, where $I$ denotes the second moment of the cross-sectional area and $\rho$ is the radius of the osculating circle. Upon approximating $\frac{1}{\rho} \approx \partial_{x}^{2} u(x)$ we obtain the linear ordinary differential equation $E I \partial_{x}^{2} u(x)=F(u(L)-u(x))$, which together with the boundary conditions $u(0)=0$ and $\partial_{x} u(0)=0$ can be solved as $u(x)=u(L)(1-$ $\cos (\sqrt{F / E I} x))$. Hence, the minimum force allowing for a non-vanishing $u(L)$ is the so-called buckling load $F=\frac{E I \pi^{2}}{4 L^{2}}$. It is the smallest load for which we expect a bending of the beam towards one side rather than a symmetric compression. The physical bifurcation associated with this buckling of beams can be reproduced in a nonlinear elasticity model. Figure 5 shows simulation results for the compression of vertical bars with height one and varying thickness $t$ (actually performed with the approximate phase-field model introduced above). The top edge of each bar is subjected to a uniformly distributed surface load such that the total resulting downward force is the same for all bars. The mechanical energy components belonging to the different configurations are shown in Figure 5, bottom, as functions of the bar thickness $t$. Apparently, down to a width of $t=\hat{t} \approx 0.28$, we seem to stay in the linearly elastic regime: The deformations $\phi$ of the beams $\mathcal{O}$ are symmetric, and $\mathcal{W}[\mathcal{O}, \phi] \approx \frac{1}{2} \mathcal{C}[\phi] \approx-\mathcal{E}[\mathcal{O}, \phi]$ as in linearized elasticity. For smaller thicknesses $t$, all energy components strongly increase, and the beams bend outwards. Indeed, the simulation parameters $L=1$, $F=0.05, E=4 \mu \frac{\mu+\lambda}{2 \mu+\lambda}=\frac{32}{3}$ (with $\mu=\lambda=4$ ) and the relation $I=\frac{t^{3}}{12}$ yield $\hat{t}=\sqrt[3]{48 F L^{2} /\left(\pi^{2} E\right)} \approx 0.2836$. Note that there is a beam width $\tilde{t}$ below which the stored elastic energy $\mathcal{W}[\mathcal{O}, \phi]$ decreases again. This behavior is linked to the observation in Section 3.2 concerning the difference between $\mathcal{W}[\mathcal{O}, \phi]$ and $\mathcal{C}[\phi]$. The thinner a beam the less bending energy is stored, and its base behaves more like a hinge so that the entire configuration resembles just a hanging, dilated rod, which absorbs relatively little elastic energy.

For the example of a compressed beam, the symmetric minimizing state apparently stops existing at $\hat{t}$. However, it is not necessarily true that the state which qualitatively corresponds to the equilibrium deformation in linearized elasticity does not persist in parallel to other (local) equilibrium states. Indeed in Figure 6 we consider a shape which emerged as an intermediate result during the relaxation of our nonlinear elasticity and phase-field model, shown top left. The structure is fixed at the bottom and subjected to a surface load at its top. As before in Figure 3, white areas correspond to stiff material while black regions represent very soft material and grey levels indicate some intermediate stiffness. If we now gradually increase the magnitude of the surface load, then we observe two families of deformations, which locally minimize the free energy $\mathcal{E}$. One of them qualitatively corresponds to the equilibrium of linearized elasticity ((a)-(e) in Fig. 6), whereas the other, buckling-type family ([f]-[g] in Fig. 6) is a particular outcome of the nonlinear elastic model. As shown in the energy diagram, the two families (a)-(e) and [f]-[j] lie on two branches, which bifurcate around a scale value 0.06 , and indeed there 

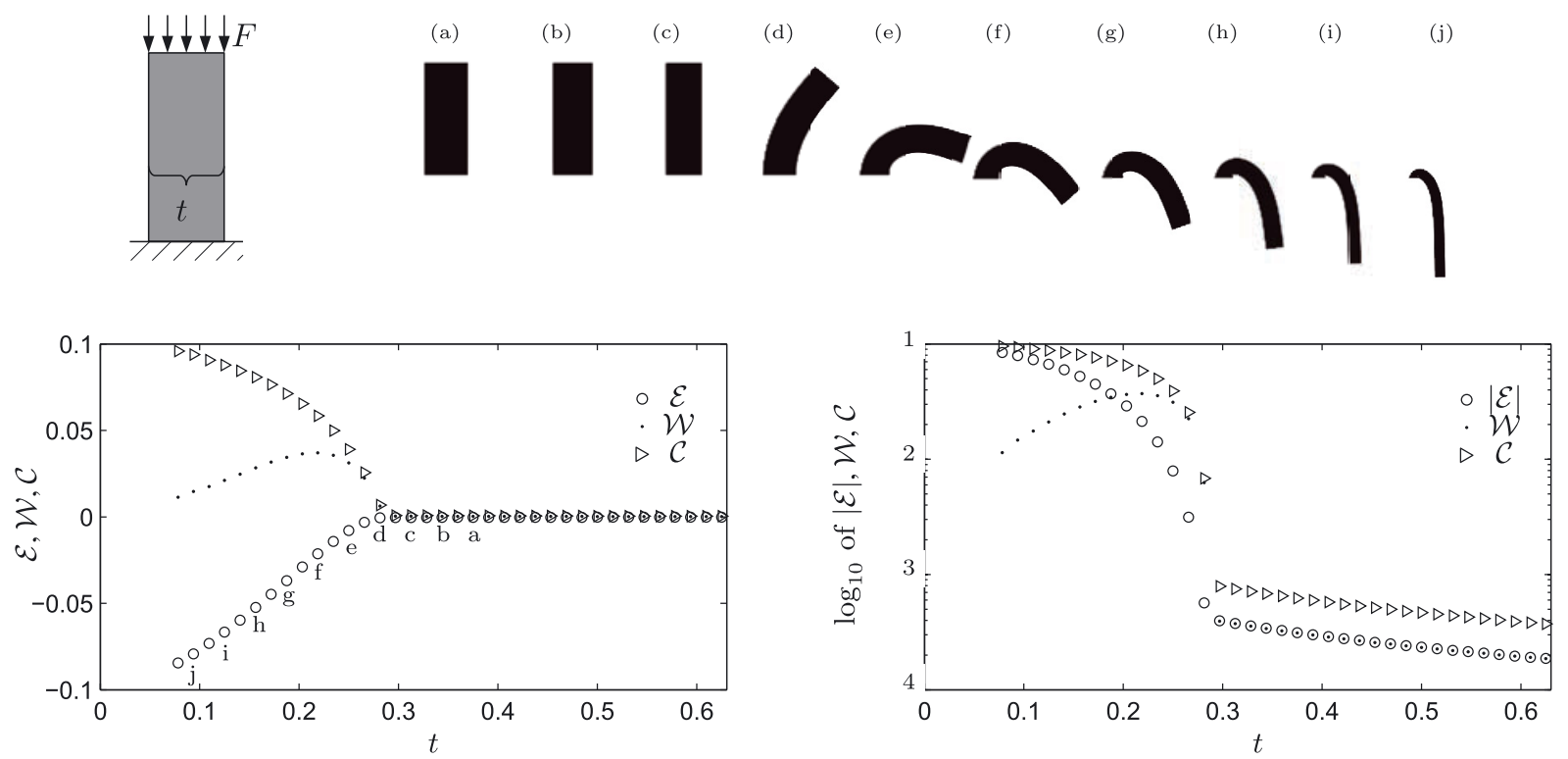

Figure 5. Buckling of beams of varying thickness and the different associated compliance components. Top: Sketch of the load configuration (left) and corresponding equilibrium deformations for beams of varying width $t$ (from $(\mathrm{a})$ to $(\mathrm{j})$ ). Bottom: $\mathcal{E}[\mathcal{O}, \phi], \mathcal{W}[\mathcal{O}, \phi]$, and $\mathcal{C}[\phi]$ for the equilibrium deformation as a function of the beam thickness $t$ (the second graph shows a logarithmic plot).

is a crossover with respect to the free energy at a scale value 0.08 , approximately corresponding to the deformations (b) and $[\mathrm{g}]$. For the computation of these two branches we use a homotopy method: starting from the deformation of linearized elasticity, we gradually increase the load and compute the corresponding deformations using the previous deformation as initial guess. At the highest load, the numerical minimization algorithm for the free energy $\mathcal{E}$ suddenly (probably due to some small perturbation) detects the different equilibrium state which we then take as initial guess to compute the second equilibrium deformations for successively decreased surface load (for the actual numerical algorithm see Sect. 6). Incidentally, deformations [f]-[j] in Figure 6 are non-physical in the sense that the material penetrates itself ( $c f$. the split view of deformation [h]). As a matter of fact, we do not impose Dirichlet boundary conditions except for the lower boundary but let the deformation relax freely. The obtained deformation appears to be locally a particular instance of the example from [12] of a (even locally) non-invertible deformation of the unit disc in $\mathbb{R}^{2}$ of the type $(r, \theta) \mapsto(r, 2 \theta)$ in polar coordinates. The existence of simultaneously existing, geometrically different local equilibria with identical energy plays a crucial role in the discussion of the existence of optimal shapes in Section 5.1.

\section{The EXISTENCE PROBlEM IN NONLINEAR ELASTIC SHAPE OPTIMIZATION}

Whether an optimal shape exists or not strongly depends on the objective we aim to minimize. In this section we first prove existence of optimizing phase fields, where in case of multiple global equilibrium deformations we always choose the one with least compliance. Likewise, we show existence of optimal shapes in a corresponding sharp-interface model. Furthermore, for a worst-case optimization, where we aim at minimizing the compliance with respect to the deformation that, among all global minimizers of the free energy, yields the largest compliance, we show that in general we cannot expect existence of an optimal shape. Finally, the behavior of the phase-field model in the limit for vanishing phase-field parameter $\varepsilon$ will be investigated. 

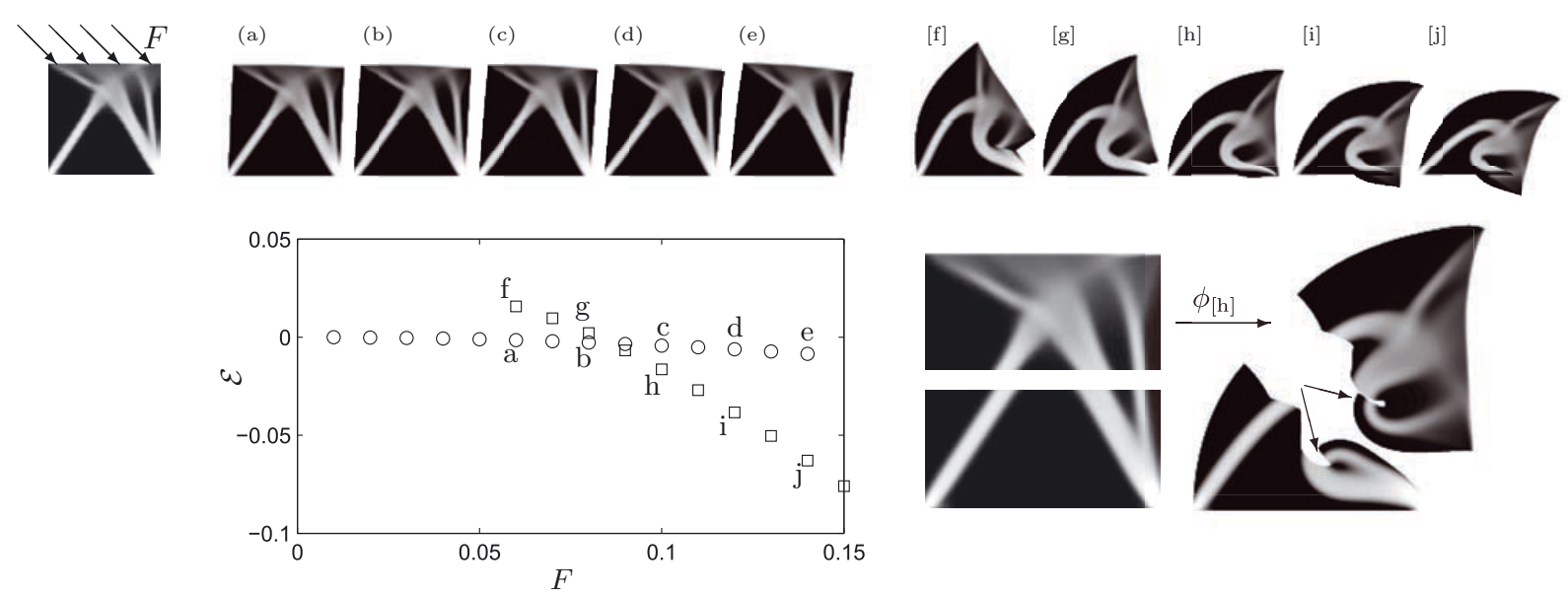

FigurE 6. Top: Sketch of the load carried by the object (left) and the resulting two families (a)-(e) and [f]-[j] of (pairwise simultaneously existing) equilibrium deformations for an increasing load. Bottom: $\mathcal{E}[\mathcal{O}, \phi]$ for the equilibrium deformations as a function of the load magnitude (left). The right figure shows deformation [h] applied to the bottom and the top half of the object, where the region of material self-penetration is marked by arrows.

\subsection{Existence of minimizers for a least compliance optimization}

We aim to establish the existence of a phase field $v$ that minimizes $\mathcal{J}_{\mathcal{W}}^{\varepsilon}[v, \phi[v]]$ or alternatively $\mathcal{J}_{\mathcal{C}}^{\varepsilon}[v, \phi[v]]$ under the constraint that $\phi[v]$ minimizes $\mathcal{E}^{\delta}[v, \cdot]$. Let us first verify some properties of the functional $\mathcal{E}^{\delta}$. In what follows we will always assume $d \in\{2,3\}$ and $\Omega \subset \mathbb{R}^{d}$ to be bounded, open, and connected with Lipschitz boundary.

Theorem 5.1 (existence of equilibrium deformations for fixed phase field). Let $v \in L^{1}(\Omega)$. If $W: \mathbb{R}^{d \times d} \rightarrow \mathbb{R}$ is polyconvex with $W(A) \geq C_{1}\|A\|_{F}^{p}-C_{2}, p>d$, and $F \in L^{1}\left(\Gamma_{N}\right)$, then the variational problem $\min _{\phi} \mathcal{E}^{\delta}[v, \phi]$ admits a minimizer in $\left\{\phi \in W^{1, p}(\Omega):\left.\phi\right|_{\Gamma_{D}}=\mathrm{id}\right\}$.

Proof. For the proof we refer to [11] or the exposition in [21]. The proof relies on the weak lower semicontinuity of the energy $\mathcal{W}^{\delta}[v, \cdot]$ for fixed phase field $v$ and makes use of the weak continuity of the cofactor and the determinant of the deformation gradient.

In the previous theorem, the phase field $v$ was fixed. However, we are interested in the impact of shape variations and thus in a variation of the shape-describing phase field. Indeed, we will need a weak lower semi-continuity result with respect to both the deformation and the phase field, as provided by the following lemma.

Lemma 5.2. For $W: \mathbb{R}^{d \times d} \rightarrow \mathbb{R}$ being polyconvex and bounded from below and for $F \in L^{1}\left(\Gamma_{N}\right)$ the functionals $\mathcal{W}^{\delta}[v, \phi]$ and $\mathcal{E}^{\delta}[v, \phi]$ are sequentially lower semi-continuous along sequences $\left(v_{i}, \phi_{i}\right)_{i \in \mathbb{N}}$ with $v_{i} \rightarrow v$ in $L^{1}(\Omega)$, $\phi_{i} \rightarrow \phi$ in $W^{1, p}(\Omega)$, and $\left(\mathcal{D} \phi_{i}, \operatorname{cof} \mathcal{D} \phi_{i}, \operatorname{det} \mathcal{D} \phi_{i}\right) \rightarrow(\mathcal{D} \phi, \operatorname{cof} \mathcal{D} \phi, \operatorname{det} \mathcal{D} \phi)$ in $L^{p}(\Omega) \times L^{q}(\Omega) \times L^{r}(\Omega)$ for $p>d$, $q, r>1$.

Proof. Due to the polyconvexity of $W$, we may write $W(A)=\bar{W}(A, \operatorname{cof} A, \operatorname{det} A)$ for a convex function $\bar{W}$. Hence we have to consider the lower semi-continuity of

$$
\left(v_{i}, \phi_{i}\right) \mapsto \int_{\Omega} \chi_{\mathcal{O}}\left(v_{i}\right) \bar{W}\left(\mathcal{D} \phi_{i}, \operatorname{cof} \mathcal{D} \phi_{i}, \operatorname{det} \mathcal{D} \phi_{i}\right) \mathrm{d} x
$$


for $i \rightarrow \infty$, which can be obtained by a straightforward adaptation of the arguments in [11] taking into account the continuity of the function $\chi_{\mathcal{O}}(\cdot)$. The lower semi-continuity of $\mathcal{C}$ is obvious, as $\phi_{i} \rightarrow \phi$ strongly in $C^{0}\left(\Gamma_{N}\right)$, and the integrand is linear in $\phi$.

Lemma 5.3. Suppose $W: \mathbb{R}^{d \times d} \rightarrow \mathbb{R}$ is polyconvex with $W(A) \geq C_{1}\|A\|_{F}^{p}-C_{2}$ for $p>d$ and $F \in L^{1}\left(\Gamma_{N}\right)$. Then, for a sequence $\left(v_{i}\right)_{i \in \mathbb{N}} \subset L^{\infty}(\Omega)$ with $\left\|v_{i}\right\|_{\infty} \leq C$ and $v_{i} \rightarrow v$ in $L^{1}(\Omega)$ one obtains

$$
\Gamma-\lim _{i \rightarrow \infty} \mathcal{E}^{\delta}\left[v_{i}, \cdot\right]=\mathcal{E}^{\delta}[v, \cdot]
$$

with respect to the weak $W^{1, p}(\Omega)$-topology.

Proof. Since the boundary integral $\mathcal{C}[\cdot]$ is just a continuous perturbation, we need to show $\Gamma$-convergence of $\mathcal{W}^{\delta}\left[v_{i}, \cdot\right]$ only.

Let $\phi_{i} \rightarrow \phi$ in $W^{1, p}(\Omega)$ with $\limsup _{i \rightarrow \infty} \mathcal{W}^{\delta}\left[v_{i}, \phi_{i}\right]<\infty$. From the growth conditions on $W$ we deduce the boundedness of $\left(\operatorname{cof} \mathcal{D} \phi_{i}, \operatorname{det} \mathcal{D} \phi_{i}\right)$ in $L^{p /(d-1)}(\Omega) \times L^{p / d}(\Omega)$ and thus - due to the reflexivity of the Lebesgue spaces - the weak convergence of a subsequence. Then, we can apply Ball's compensated compactness result [11] to obtain $\left(\mathcal{D} \phi_{i}, \operatorname{cof} \mathcal{D} \phi_{i}, \operatorname{det} \mathcal{D} \phi_{i}\right) \rightarrow(\mathcal{D} \phi, \operatorname{cof} \mathcal{D} \phi, \operatorname{det} \mathcal{D} \phi)$ in $L^{p}(\Omega) \times L^{p /(d-1)}(\Omega) \times L^{p / d}(\Omega)$. The previous lemma then yields the liminf-inequality, that is, $\liminf _{i \rightarrow \infty} \mathcal{W}^{\delta}\left[v_{i}, \phi_{i}\right] \geq \mathcal{W}^{\delta}[v, \phi]$.

For the $\lim$ sup-inequality, note that $\mathcal{W}^{\delta}\left[v_{i}, \phi\right] \rightarrow \mathcal{W}^{\delta}[v, \phi]$. Otherwise there would be a $\rho>0$ and a subsequence $\left(v_{j}\right)_{j \in J \subset \mathbb{N}}$, such that $\left|\mathcal{W}^{\delta}\left[v_{j}, \phi\right]-\mathcal{W}^{\delta}[v, \phi]\right|>\rho$ for all $j \in J$. Since $v_{j} \rightarrow v$ in $L^{1}(\Omega)$, we can furthermore assume that $v_{j} \rightarrow v$ pointwise almost everywhere as $j \rightarrow \infty$ in $J$. The integrand of $\mathcal{W}^{\delta}\left[v_{j}, \phi\right]$ is bounded from above by $\left((1-\delta) \frac{1}{4}(C+1)^{2}+\delta\right) W(\mathcal{D} \phi)$ and converges pointwise to $\chi_{\mathcal{O}}(v) W(\mathcal{D} \phi)$. By the dominated convergence theorem, we obtain $\mathcal{W}^{\delta}\left[v_{j}, \phi\right] \rightarrow \mathcal{W}^{\delta}[v, \phi]$ as $j \rightarrow \infty$ in $J$, which is a contradiction. Hence, for the recovery sequence $\phi_{i}=\phi$ for all $i \in \mathbb{N}$, we obtain $\lim \sup _{i \rightarrow \infty} \mathcal{W}^{\delta}\left[v_{i}, \phi_{i}\right]=\mathcal{W}^{\delta}[v, \phi]$ which proves the lim sup-inequality.

Based on these preliminaries we are now able to show the existence of minimizing phase fields $v$ for the shape optimization problem under the constraint that for fixed phase field $v$ the deformation $\phi$ is in the set of global minimizers of the elastic free energy.

Theorem 5.4 (existence of optimal shape-encoding phase fields). Suppose $W: \mathbb{R}^{d \times d} \rightarrow \mathbb{R}$ is polyconvex with $W(A) \geq C_{1}\|A\|_{F}^{p}-C_{2}$ for $p>d$ and $F \in L^{1}\left(\Gamma_{N}\right)$. Furthermore, consider phase fields $v \in W^{1,2}(\Omega)$ with $-1 \leq v \leq 1$. Then the variational problem $\min _{v} \mathcal{J}_{\mathcal{G}}^{\varepsilon}[v, \phi]$ with $\mathcal{G}$ being $\mathcal{W}, \mathcal{C}$, or $\mathcal{D}$ admits a minimizer under the constraint $\phi \in \mathfrak{m}[v]$, where $\mathfrak{m}[v]$ is the set of minimizing deformations of $\mathcal{E}^{\delta}[v, \cdot]$ in $\left\{\phi \in W^{1, p}(\Omega):\left.\phi\right|_{\Gamma_{D}}=\mathrm{id}\right\}$.

Proof. At first, note that for $v$ in $\left\{v \in W^{1,2}(\Omega):-1 \leq v \leq 1\right\}$ the energy $\mathcal{E}^{\delta}[v$, id] is uniformly bounded from above by a constant $\overline{\mathcal{E}}<\infty$. Consequently, $\mathcal{E}^{\delta}[v, \phi] \leq \overline{\mathcal{E}}$ for all $v$ and $\phi \in \mathfrak{m}[v]$. Also, due to the embedding $W^{1, p}(\Omega) \hookrightarrow C^{0}(\bar{\Omega})$ we have $|\mathcal{C}[\phi]| \leq C\|\phi\|_{W^{1, p}(\Omega)}$ for some $C>0$. Together with the growth conditions on $W$ and $\mathcal{E}^{\delta}[v, \phi] \leq \overline{\mathcal{E}}$ we deduce that $\|\phi\|_{W^{1, p}(\Omega)}$ is bounded and thus $|\mathcal{C}[\phi]| \leq \overline{\mathcal{C}}$ and consequently also $\left|\mathcal{W}^{\delta}[v, \phi]\right| \leq \overline{\mathcal{W}}$ for $\overline{\mathcal{W}}, \overline{\mathcal{C}}<\infty$. Clearly, $\mathcal{J}_{\mathcal{G}}^{\varepsilon}[v, \phi]$ with $\phi \in \mathfrak{m}[v]$ is then uniformly bounded from below by some constant for all admissible $v \in W^{1,2}(\Omega)$.

Now, we consider a minimizing sequence $\left(v_{i}\right)_{i \in \mathbb{N}}$ in $\left\{v \in W^{1,2}(\Omega):-1 \leq v \leq 1\right\}$. Due to the weak $W^{1,2}(\Omega)$ coercivity of $\mathcal{J}_{\mathcal{G}}^{\varepsilon}$ with respect to the phase field (by virtue of the regularization $\mathcal{L}_{\mathrm{MM}}^{\varepsilon}[v]$ and the reflexivity of $\left.W^{1,2}(\Omega)\right)$ there is $v \in W^{1,2}(\Omega)$ with $-1 \leq v \leq 1$ such that $v_{i} \rightarrow v$ in $W^{1,2}(\Omega)$ (after extraction of a subsequence), and thus $v_{i}$ converges strongly to $v$ in $L^{1}(\Omega)$ as $i \rightarrow \infty$.

Next, let $\phi\left[v_{i}\right] \in \mathfrak{m}\left[v_{i}\right]$ denote one sequence of deformations associated with the minimizing sequence $\left(v_{i}\right)_{i \in \mathbb{N}}$. Then, due to the uniform boundedness of $\phi\left[v_{i}\right]$ in $W^{1, p}(\Omega)$ (by the growth condition on $W$ ) and the reflexivity of $W^{1, p}(\Omega)$, there is a deformation $\phi \in W^{1, p}(\Omega)$ with $\phi(x)=x$ for $x \in \Gamma_{D}$ such that $\phi\left[v_{i}\right] \rightarrow \phi$ (after extracting a subsequence). Since for fixed $\delta>0$ the free energy $\mathcal{E}^{\delta}\left[v_{i}, \cdot\right]$ is equi-mildly coercive, Lemma 5.3 implies $\phi \in \mathfrak{m}[v]$. Here, note that the $\Gamma$-limit is consistent with the Dirichlet boundary conditions at $\Gamma_{D}$.

Finally, $\mathcal{J}_{\mathcal{G}}^{\varepsilon}\left[v_{i}, \phi\left[v_{i}\right]\right]$ is sequentially weakly lower semi-continuous as $v_{i} \rightarrow v$ in $W^{1,2}(\Omega)$ and $\phi\left[v_{i}\right] \rightarrow \phi$ in $W^{1, p}(\Omega)$. In fact, the lower semi-continuity of $\mathcal{L}_{\mathrm{MM}}^{\varepsilon}\left[v_{i}\right]$ and $\mathcal{V}\left[v_{i}\right]$ is obvious as their integrands are convex in $\nabla v$ 
and continuous in $v$. Furthermore, the $\Gamma$-convergence of $\mathcal{E}^{\delta}$ stated in Lemma 5.3 ensures that $\mathcal{E}^{\delta}\left[v_{i}, \phi\left[v_{i}\right]\right] \rightarrow$ $\left.\mathcal{E}^{\delta}[v, \phi]\right]$ for $i \rightarrow \infty$. Likewise, by the compact embedding of $W^{1, p}(\Omega)$ in $L^{\infty}\left(\Gamma_{N}\right), \phi\left[v_{i}\right] \rightarrow \phi$ strongly in $L^{\infty}\left(\Gamma_{N}\right)$ so that $\mathcal{C}\left[\phi\left[v_{i}\right]\right]$ converges to $\mathcal{C}[\phi]$. Finally, as in the proof of the previous lemma, we may assume $\left(\mathcal{D} \phi\left[v_{i}\right], \operatorname{cof} \mathcal{D} \phi\left[v_{i}\right], \operatorname{det} \mathcal{D} \phi\left[v_{i}\right]\right) \rightarrow(\mathcal{D} \phi, \operatorname{cof} \mathcal{D} \phi, \operatorname{det} \mathcal{D} \phi)$ so that $\mathcal{W}^{\delta}[v, \phi] \leq \liminf _{i \rightarrow \infty} \mathcal{W}^{\delta}\left[v_{i}, \phi\left[v_{i}\right]\right]$ follows from Lemma 5.2. From the above, $\mathcal{J}_{\mathcal{G}}^{\varepsilon}[v, \phi] \leq \liminf _{i \rightarrow \infty} \mathcal{J}_{\mathcal{G}}^{\varepsilon}\left[v_{i}, \phi\left[v_{i}\right]\right]$ with $\phi \in \mathfrak{m}[v]$, and hence $v$ is a minimizer.

Remark 5.5. In contrast to other phase-field models, the constraint $-1 \leq v \leq 1$ does not follow from a straightforward comparison argument. Indeed, $\mathcal{E}^{\delta}[v, \phi] \geq \mathcal{E}^{\delta}[\max (0, \min (1, v))$, $\bar{\phi}]$ does not hold in general. Hence, to be able to apply Lemma 5.3 we have to impose an $L^{\infty}$-bound for the phase field $v$ as a constraint. As an alternative, we might consider a different function $\chi_{\mathcal{O}}(\cdot)$, which is continuous and a priori uniformly bounded on $\mathbb{R}$, for example

$$
\chi_{\mathcal{O}}(v):=\min \left(1, \frac{1}{4}(v+1)^{2}\right) .
$$

In our numerical simulations, however, it was not necessary to cut off $\chi_{\mathcal{O}}(v)$ or to implement an $L^{\infty}$-bound for $v$.

The above existence result still holds if instead of describing shapes via phase fields $v \in W^{1,2}(\Omega)$ we consider the sharp-interface case and represent shapes by functions of bounded variation $v \in B V(\Omega,\{-1,1\})$, as shown in the following theorem. In that case, the shape perimeter is expressed via the total variation of $v$.

Theorem 5.6 (existence of optimal shapes). Suppose $W: \mathbb{R}^{d \times d} \rightarrow \mathbb{R}$ is polyconvex with $W(A) \geq C_{1}\|A\|_{F}^{p}-C_{2}$ for $p>d$ and $F \in L^{1}\left(\Gamma_{N}\right)$. Consider the functional

$$
\mathcal{J}_{\mathcal{G}}^{0}[v, \phi]:=\mathcal{R}+\nu \mathcal{V}[v]+\frac{\eta}{2}|v|_{\mathrm{TV}(\Omega)}
$$

with $\mathcal{R}$ being $2 \mathcal{W}^{\delta}[v, \phi], \mathcal{C}[\phi]$, or $-2 \mathcal{E}^{\delta}[v, \phi]$ (for $\mathcal{G}=\mathcal{W}, \mathcal{C}$, or $\mathcal{D}$, respectively) and $|\cdot| \operatorname{TV}(\Omega)$ denoting the total variation. Then the variational problem $\min _{v} \mathcal{J}_{\mathcal{G}}^{0}[v, \phi]$ admits a minimizer in $B V(\Omega,\{-1,1\})$ under the constraint $\phi \in \mathfrak{m}[v]$, where $\mathfrak{m}[v]$ is defined as in Theorem 5.4 .

Proof. The proof follows along the same lines as that of Theorem 5.4. The boundedness of the cost functional from below is obtained just as in the phase-field case. We consider a minimizing sequence $\left(v_{i}\right)_{i \in \mathbb{N}} \subset$ $B V(\Omega,\{-1,1\})$, which is bounded due to the total variation regularization in $\mathcal{J}_{\mathcal{G}}^{0}$. Hence, there is a weak-* convergent subsequence, again denoted $\left(v_{i}\right)_{i \in \mathbb{N}}$, with weak- $*$ limit $v \in B V(\Omega,\{-1,1\})$. We deduce $v_{i} \rightarrow v$ strongly in $L^{1}(\Omega)$, and as in the proof of Theorem 5.4 we can find a sequence of equilibrium deformations $\phi\left[v_{i}\right] \in \mathfrak{m}\left[v_{i}\right]$ and $\phi \in \mathfrak{m}[v]$ such that $\phi\left[v_{i}\right] \rightarrow \phi$ in $W^{1, p}(\Omega)$. The lower semi-continuity of $\mathcal{J}_{\mathcal{G}}^{0}\left[v_{i}, \phi\left[v_{i}\right]\right]$ as $v_{i} \stackrel{*}{\rightarrow} v$ in $B V(\Omega,\{-1,1\})$ and $\phi\left[v_{i}\right] \rightarrow \phi$ in $W^{1, p}(\Omega)$ then follows as before, noting the lower semi-continuity of $\left|v_{i}\right| \operatorname{TV}(\Omega)$.

Remark 5.7. The growth condition on the elastic deformation energy density $W$ has only been chosen for simplicity. As already stated earlier, one can just as well impose growth conditions of the form $W(A) \geq$ $C_{1}\left(\|A\|_{F}^{p}+\|\operatorname{cof} A\|_{F}^{q}+|\operatorname{det} A|^{r}\right)-C_{2}$, where $p \leq d$ with appropriately chosen $q$ and $r$ [28]. In this case, however, stronger restrictions on the load $F ;$ e.g. $F \in L^{p^{\prime}}\left(\Gamma_{N}\right)$ with $\frac{1}{p^{\prime}}+\frac{1}{p}=1$, are needed.

\subsection{Non-existence of minimizers in a worst case scenario}

For the sake of simplicity, the following discussion refers to the phase-field model from Section 3.3. In fact, we underpin our argumentation with numerical results for the phase-field model. However, the same arguments also apply to the sharp-interface case from Theorem 5.6.

The result from the previous section only states that there is a phase field $v$ and one equilibrium deformation $\phi \in \mathfrak{m}[v]$ such that $\mathcal{J}_{\mathcal{G}}^{\varepsilon}[v, \phi]$ is minimal. There are possibly more equilibrium deformations $\tilde{\phi} \in \mathfrak{m}[v]$ for which $\mathcal{J}_{\mathcal{G}}^{\varepsilon}[v, \tilde{\phi}]>\mathcal{J}_{\mathcal{G}}^{\varepsilon}[v, \phi]$ in case $\mathcal{G}$ is either $\mathcal{W}$ or $\mathcal{C}$. Such worst case deformations are expected to represent stronger 

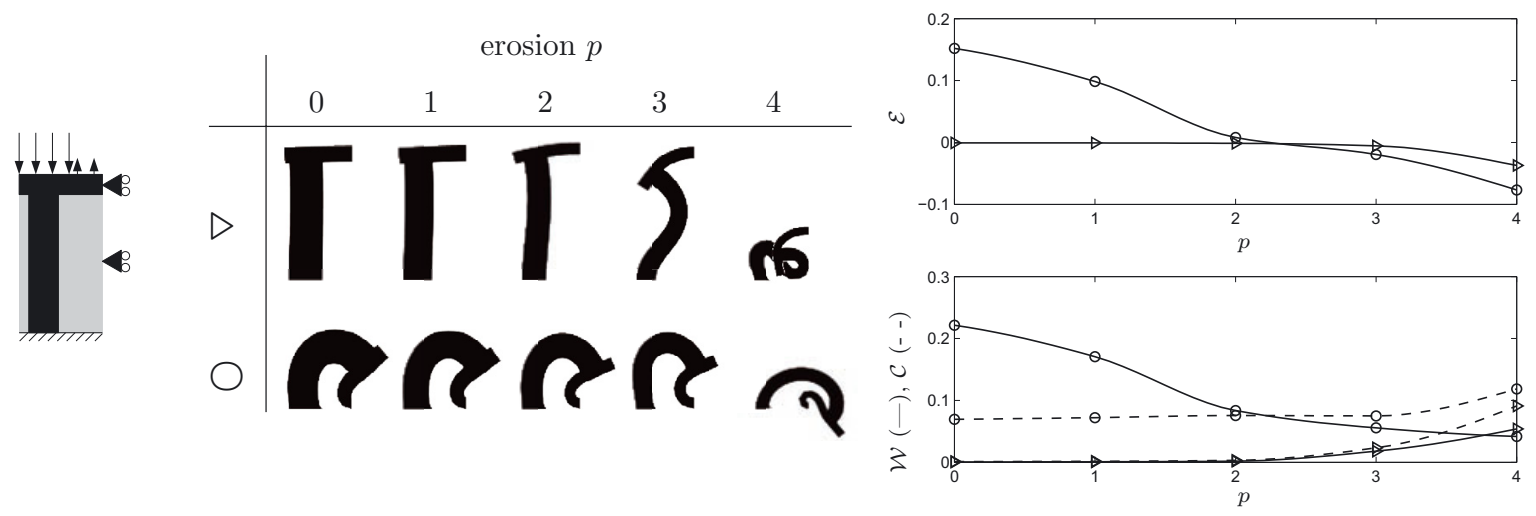

FiguRE 7. Left: Sketch of the shape optimization problem under consideration. Black represents a stiff, grey a very soft material. Middle: Equilibrium deformations for shapes of different thickness, starting from a reference shape on the left and gradually eroding the stiff material to the right (the soft material is not displayed). The top and bottom row represent two different equilibria. Right: Energy components of the different configurations.

strains ( $c f$. Fig. 6). For this reason, it might be more interesting to actually consider the (worst case) objective functional

$$
\overline{\mathcal{J}_{\mathcal{G}}^{\varepsilon}}[v]:=\sup _{\phi \in \mathfrak{m}[v]} \mathcal{J}_{\mathcal{G}}^{\varepsilon}[v, \phi] .
$$

However, minimizers for $\overline{\mathcal{J}_{\mathcal{G}}^{\varepsilon}}$ seem not to exist in general as the following example illustrates.

We would like to optimize the structure in Figure 7, left. It is composed of two different materials: The vertical pillar and the crossbeam consist of a stiff material, while the material below the crossbeam, right and left of the vertical beam, is very soft. The object is clamped at its bottom and subjected to a surface load from the top. Its right-most edge can move freely in vertical direction, but is fixed in horizontal direction. Now, instead of optimizing the structure within the set of all possible shapes, we will only consider a simple, one-dimensional subset which is generated by eroding the vertical and horizontal beam of the original shape depicted in the sketch. That is, we try to find just the optimal thickness of the stiff components.

The underlying idea of this example is the following:We seek a configuration with two simultaneously existing equilibrium deformations, which is given by the compressed vertical beam that can buckle to its left or right side. One configuration should be initially preferred (that is, be the global minimizer of $\mathcal{E}$ in the case of rather thick and thus stiff structures), while the other should take over at some point if the structure becomes less stiff and is deformed more strongly. The initial preference for rightward buckling is achieved by adding the slight upward traction at the top right of the shape. If the pillar buckles so strongly that the soft material between it and the right wall is completely compressed, the structure stiffens (due to the additional support by the wall), and hence the leftward buckling will at some point yield less free energy and become the global minimizer of $\mathcal{E}$. Here, the soft material between the pillar and the wall obviously just serves to transmit a force from the wall onto the pillar. In fact, this soft material in our numerical setup just represents a means to transmit forces which increase as the pillar gets close to the right wall.

The top and bottom row of the table in Figure 7 depict two different equilibrium deformations for the prescribed loading (that is, stable deformations which are local minimizers of $\mathcal{E}$ ), where each column belongs to a different object thickness: From left to right, the original shape (whose vertical pillar has a width of six length units) is eroded by zero up to four length units (in the underlying computations, one length unit actually corresponds to a grid cell). The corresponding total free energy $\mathcal{E}$ as well as the stored elastic energy $\mathcal{W}$ and the change in external potential $\mathcal{C}$ are shown in the right graph, where triangles and circles represent the energies 

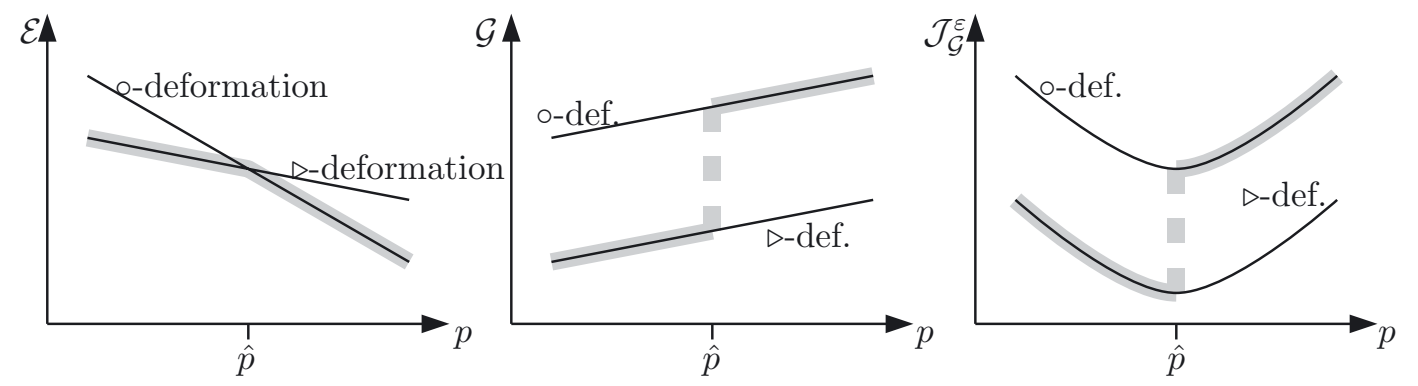

FigurE 8. Qualitative sketch of the energies belonging to the $\circ$ - and $\triangleright$-deformations from Figure 7. The curves belonging to the global equilibrium deformation (the global minimizer of $\mathcal{E}$ ) are highlighted in grey. At $p=\hat{p}$, the global equilibrium deformation switches from $\phi_{\triangleright p}$ to $\phi_{\text {op }}$, which is associated with a jump in $\mathcal{W}$ or in $\mathcal{C}$ and a corresponding jump in $\overline{\mathcal{J}_{\mathcal{G}}^{\varepsilon}}$ (thick grey curve in the rightmost graph).

of the configurations in the upper and lower table row, respectively. For ease of reference, we shall denote the deformations by $\phi_{\triangleright p}$ and $\phi_{\circ p}, 0 \leq p \leq 4$.

We start the optimization from the thickest shape (left end of table and graph). The equilibrium deformation which globally minimizes $\mathcal{E}$ is obviously given by $\phi_{\triangleright 0}$ (compare Fig. 7, right). Assume that the volume penalty parameter $\nu$ is chosen large enough so that the objective functional $\overline{\mathcal{J}_{\mathcal{G}}^{\varepsilon}}$ decreases for increasing erosion parameter $p$. Then $\phi_{\triangleright p}$ stays the global equilibrium deformation up to a point $p=\hat{p}$ between 2 and 3 , where suddenly $\phi_{\mathrm{op}}$ takes over as the global equilibrium deformation. At this point, the objective functional jumps discontinuously to a higher value, since $\mathcal{W}$ as well as $\mathcal{C}$ are larger for the deformations $\phi_{\circ p}$ than for $\phi_{\triangleright p}$. Hence, if the weight $\nu$ of the volume term is chosen such that - neglecting the existence of the equilibrium deformations $\phi_{\circ p}$ - the optimal thickness would lie exactly at the crossing point $\hat{p}$, then there will be no minimizer ( $c f$. the qualitative sketch in Fig. 8). Indeed, from the left we can get arbitrarily close to $\hat{p}$ and thus the objective functional gets arbitrarily close to its infimum value, but we cannot reach it since at $\hat{p}$, the cost functional suddenly jumps up.

A nice feature of the above example is that the equilibrium deformation $\phi_{\circ p}$ initially yields a positive value of $\mathcal{E}$ or, in other words, that the stored elastic energy $\mathcal{W}$ is larger than the external potential change $\mathcal{C}$. This is associated with a self-locking mechanism: Even without loads, a deformation of the type $\phi_{\text {op }}$ would be stable and the object cannot be deformed into a less strained state without intermediately increasing the strain.

Having presented this example, the question arises naturally whether one should actually go even further and consider $\mathcal{W}$ and $\mathcal{C}$ for all local equilibrium deformations, that is, extend the set $\mathfrak{m}[v]$ to the set of all local minimizers of $\mathcal{E}[v, \cdot]$. However, this complicates the system even further, and one would also have to pay attention to exclude unphysical states that, for example, imply a local reversion of orientation such as shown in Figure 9 for the example above.

Remark 5.8. So far we have investigated the compliance functionals $\mathcal{J}_{\mathcal{W}}^{\varepsilon}$ and $\mathcal{J}_{\mathcal{C}}^{\varepsilon}$. For the compliance functional

$$
\mathcal{J}_{\mathcal{D}}^{\varepsilon}[v, \phi]=2 \mathcal{C}[\phi]-2 \mathcal{W}^{\delta}[v, \phi]+\nu \mathcal{V}[v]+\eta \mathcal{L}_{\mathrm{MM}}^{\varepsilon}[v]=-2 \mathcal{E}^{\delta}[v, \phi]+\nu \mathcal{V}[v]+\eta \mathcal{L}_{\mathrm{MM}}^{\varepsilon}[v]
$$

which represents the dissipation associated with the transition from the unstressed state to the equilibrium deformation, existence of minimizing phase fields can be established also for the worst case scenario

$$
\overline{\mathcal{J}_{\mathcal{D}}^{\varepsilon}}[v]:=\sup _{\phi \in \mathfrak{m}[v]} \mathcal{J}_{\mathcal{D}}^{\varepsilon}[v, \phi]
$$

Indeed, we observe that $\sup _{\phi \in \mathfrak{m}[v]}\left(-2 \mathcal{E}^{\delta}[v, \phi]\right)=-2 \inf _{\phi \in \mathfrak{m}[v]} \mathcal{E}^{\delta}[v, \phi]=-2 \inf \left\{\mathcal{E}^{\delta}[v, \phi] \mid \phi \in W^{1, p}, \phi=\mathrm{id}\right.$ on $\left.\Gamma_{D}\right\}$. 


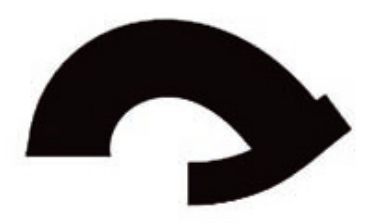

Figure 9. Local equilibrium deformation for the mechanical problem in Figure 7 which reverses the orientation of the crossbeam and cannot be reached mechanically starting from the configuration in Figure 7, left.

\subsection{Phase-field model behavior in the sharp-interface limit for $\varepsilon \rightarrow 0$}

In the phase-field model, we have so far assumed the phase-field parameter $\varepsilon$ to be fixed. However, we are actually interested in the limit case of sharp interfaces, for which existence of optimal shapes has already been shown in Theorem 5.6 and which we hope to recover when we let $\varepsilon \rightarrow 0$. Unfortunately, the non-uniqueness of the equilibrium deformation prevents us from proving a general $\Gamma$-convergence result: It might theoretically happen that - as $\varepsilon$ reaches zero and the phase-field interface gets ultimately sharp - suddenly an additional equilibrium deformation occurs which results in a sudden increase or decrease of the objective functional value. For this reason, we can only state the following two weaker results.

Let us define for $v \in W^{1,2}(\Omega)$ with $-1 \leq v \leq 1$ the compliance functionals $\mathcal{J}_{\mathcal{G}}^{\varepsilon}[v]:=\inf _{\phi \in \mathfrak{m}[v]} \mathcal{J}_{\mathcal{G}}^{\varepsilon}[v, \phi]$ and as above $\overline{\mathcal{J}_{\mathcal{G}}^{\varepsilon}}[v]:=\sup _{\phi \in \mathfrak{m}[v]} \mathcal{J}_{\mathcal{G}}^{\varepsilon}[v, \phi]$ for $\mathcal{G}=\mathcal{W}$ or $\mathcal{G}=\mathcal{C}$ and extend these definitions by $\infty$ on $L^{1}(\Omega)$. Furthermore, define on $L^{1}(\Omega)$

$$
\begin{aligned}
& \underline{\mathcal{J}_{\mathcal{G}}^{0}}[v]:= \begin{cases}\inf _{\phi \in \mathfrak{m}[v]} \mathcal{G}[v, \phi]+\nu \mathcal{V}[v]+\frac{\eta}{2}|v|_{\mathrm{TV}(\Omega)}, & v \in B V(\Omega,\{-1,1\}) \\
\infty, & \text { else, }\end{cases} \\
& \overline{\mathcal{J}_{\mathcal{G}}^{0}}[v]:= \begin{cases}\sup _{\phi \in \mathfrak{m}[v]} \mathcal{G}[v, \phi]+\nu \mathcal{V}[v]+\frac{\eta}{2}|v|_{\mathrm{TV}(\Omega)}, & v \in B V(\Omega,\{-1,1\}) \\
\infty, & \text { else, }\end{cases}
\end{aligned}
$$

where $|\cdot|_{\mathrm{TV}(\Omega)}$ denotes the total variation.

Theorem 5.9. Under the conditions of Theorem 5.4, we have

$$
\Gamma-\liminf _{\varepsilon \rightarrow 0} \underline{\mathcal{J}_{\mathcal{G}}^{\varepsilon}} \geq \underline{\mathcal{J}_{\mathcal{G}}^{0}}
$$

with respect to the $L^{1}(\Omega)$-topology.

Proof. Let $v_{\varepsilon} \rightarrow v$ in $L^{1}(\Omega)$ as $\varepsilon \rightarrow 0$, then obviously $\mathcal{V}\left[v_{\varepsilon}\right] \rightarrow \mathcal{V}[v]$. Furthermore,

$$
\liminf _{\varepsilon \rightarrow 0} \mathcal{L}_{\mathrm{MM}}^{\varepsilon}\left[v_{\varepsilon}\right] \geq \begin{cases}\frac{1}{2}|v|_{\mathrm{TV}(\Omega)}, & v \in B V(\Omega,\{-1,1\}) \\ \infty, & \text { else }\end{cases}
$$

as already discussed in Section 3.3 (cf. also [14]). Finally, either $\lim \inf _{\varepsilon \rightarrow 0} \inf _{\phi \in \mathfrak{m}\left[v_{\varepsilon}\right]} \mathcal{G}\left[v_{\varepsilon}, \phi\right]=\infty$, in which case there is nothing left to prove, or there is a sequence $\left(\varepsilon_{i}\right)_{i \in \mathbb{N}}$ with $\varepsilon_{i} \rightarrow 0$ as $i \rightarrow \infty$ and a sequence $\phi_{i}$ with $\phi_{i} \in \mathfrak{m}\left[v_{\varepsilon_{i}}\right]$ such that

$$
\lim _{i \rightarrow \infty} \mathcal{G}\left[v_{\varepsilon_{i}}, \phi_{i}\right]=\liminf _{\varepsilon \rightarrow 0} \inf _{\phi \in \mathfrak{m}\left[v_{\varepsilon}\right]} \mathcal{G}\left[v_{\varepsilon}, \phi\right]<\infty .
$$

From Lemma 5.3 we deduce that

$$
\Gamma-\lim _{i \rightarrow \infty} \mathcal{E}^{\delta}\left[v_{\varepsilon_{i}}, \cdot\right]=\mathcal{E}^{\delta}[v, \cdot]
$$


Furthermore, applying the same arguments as in Theorem 5.4 we obtain that $\left(\phi_{i}\right)_{i \in \mathbb{N}}$ is uniformly bounded in $W^{1, p}(\Omega)$. Hence, for a subsequence we get $\phi_{i} \rightarrow \phi$ in $W^{1, p}(\Omega)$ for some $\phi \in \mathfrak{m}[v]$ and $\mathcal{E}^{\delta}\left[v_{\varepsilon_{i}}, \phi_{i}\right] \rightarrow \mathcal{E}^{\delta}[v, \phi]$. Also, $\mathcal{C}\left[\phi_{i}\right] \rightarrow \mathcal{C}[\phi]$ due to the continuity of $\mathcal{C}$ and thus also $\mathcal{W}^{\delta}\left[v_{\varepsilon_{i}}, \phi_{i}\right]=\mathcal{E}^{\delta}\left[v_{\varepsilon_{i}}, \phi_{i}\right]+\mathcal{C}\left[\phi_{i}\right] \rightarrow \mathcal{E}^{\delta}[v, \phi]+\mathcal{C}[\phi]=\mathcal{W} \delta[v, \phi]$ so that

$$
\liminf _{\varepsilon \rightarrow 0} \inf _{\phi \in \mathfrak{m}\left[v_{\varepsilon}\right]} \mathcal{G}\left[v_{\varepsilon}, \phi\right]=\lim _{i \rightarrow \infty} \mathcal{G}\left[v_{\varepsilon_{i}}, \phi_{i}\right]=\mathcal{G}[v, \phi] \geq \inf _{\phi \in \mathfrak{m}[v]} \mathcal{G}[v, \phi]
$$

which altogether yields the desired result.

Theorem 5.10. Under the conditions of Theorem 5.4, we have

$$
\Gamma-\limsup _{\varepsilon \rightarrow 0} \overline{\mathcal{J}_{\mathcal{G}}^{\varepsilon}} \leq \overline{\mathcal{J}_{\mathcal{G}}^{0}}
$$

with respect to the $L^{1}(\Omega)$-topology.

Proof. Let $v_{\varepsilon} \rightarrow v$ be a recovery sequence in $L^{1}(\Omega)$ with respect to the $\Gamma$-convergence of $\mathcal{L}_{\mathrm{MM}}^{\varepsilon}[14]$, for which we obtain

$$
\limsup _{\varepsilon \rightarrow 0} \mathcal{L}_{\mathrm{MM}}^{\varepsilon}\left[v_{\varepsilon}\right] \leq \begin{cases}\frac{1}{2}|v|_{\mathrm{TV}(\Omega)}, & v \in B V(\Omega,\{-1,1\}) \\ \infty, & \text { else }\end{cases}
$$

As before, we have $\mathcal{V}\left[v_{\varepsilon}\right] \rightarrow \mathcal{V}[v]$. Finally, as in the previous proof, there are sequences $\varepsilon_{i}$ and $\phi_{i}$ with $\varepsilon_{i} \rightarrow 0$, $\phi_{i} \in \mathfrak{m}\left[v_{\varepsilon_{i}}\right]$, and $\phi_{i} \rightarrow \phi$ for some $\phi \in \mathfrak{m}[v]$ such that

$$
\limsup _{\varepsilon \rightarrow 0} \sup _{\phi \in \mathfrak{m}\left[v_{\varepsilon}\right]} \mathcal{G}\left[v_{\varepsilon}, \phi\right]=\lim _{i \rightarrow \infty} \mathcal{G}\left[v_{\varepsilon_{i}}, \phi_{i}\right]=\mathcal{G}[v, \phi] \leq \sup _{\phi \in \mathfrak{m}[v]} \mathcal{G}[v, \phi]
$$

which concludes the proof.

If for a given phase field $v$ there is just one single unique equilibrium deformation, then, obviously, $\overline{\mathcal{J}_{\mathcal{G}}^{0}}[v]=$ $\underline{\mathcal{J}_{\mathcal{G}}^{0}}[v]$, which immediately implies following corollary.

Corollary 5.11. Let the conditions of Theorem 5.4 hold, and let $v \in B V(\Omega,\{-1,1\})$ be given. If the equilibrium deformation is unique, that is, $\mathfrak{m}[v]=\{\phi[v]\}$ for a unique $\phi[v] \in W^{1, p}(\Omega)$, then the $\Gamma$-limit of $\overline{\mathcal{J}_{\mathcal{G}}^{\varepsilon}}$ and $\mathcal{J}_{\mathcal{G}}^{\varepsilon}$ for $\varepsilon \rightarrow 0$ with respect to the $L^{1}(\Omega)$-topology is defined at $v$ and is given by

$$
\overline{\mathcal{J}_{\mathcal{G}}^{0}}[v]=\underline{\mathcal{J}_{\mathcal{G}}^{0}}[v]
$$

As mentioned earlier, since the equilibrium deformation in general is not unique, we cannot state a general $\Gamma$-convergence result. However, note that the above results also hold with the obvious modifications in the case of linearized elasticity, that is, for

$$
\mathcal{W}^{\delta}[v, \phi]=\mathcal{W}^{\delta, \operatorname{lin}}[v, \phi]:=\int_{\Omega}\left((1-\delta) \chi_{\mathcal{O}}(v)+\delta\right) \frac{1}{2} \mathbf{C} \epsilon[\phi-\mathrm{id}]: \epsilon[\phi-\mathrm{id}] \mathrm{d} x
$$

with a symmetric positive definite elasticity tensor $\mathbf{C}$ and $\epsilon[u]=\frac{1}{2}\left(\mathcal{D} u+\mathcal{D} u^{\mathrm{T}}\right)$. In this case, where the choices $\mathcal{G}=\mathcal{W}$ or $\mathcal{D}$ are equivalent to $\mathcal{G}=\mathcal{C}$ as already discussed, we actually do obtain $\Gamma$-convergence of the objective functional. 
Corollary 5.12. For $\mathcal{W}^{\delta}=\mathcal{W}^{\delta, \text { lin }}$ and $F \in L^{2}\left(\Gamma_{N}\right)$, we have

$$
\Gamma-\lim _{\varepsilon \rightarrow 0} \overline{\mathcal{J}_{\mathcal{C}}^{\varepsilon}}=\Gamma-\lim _{\varepsilon \rightarrow 0} \underline{\mathcal{J}_{\mathcal{C}}^{\varepsilon}}=\overline{\mathcal{J}_{\mathcal{C}}^{0}}=\underline{\mathcal{J}_{\mathcal{C}}^{0}}
$$

with respect to the $L^{1}(\Omega)$-topology.

Proof. By Korn's first inequality, $\mathcal{W}^{\delta, \operatorname{lin}}[v, \cdot]$ is coercive on $\left\{\phi \in W^{1,2}(\Omega):\left.\phi\right|_{\Gamma_{D}}=\right.$ id $\}$; furthermore, it is bounded so that the Lax-Milgram lemma implies the existence of a unique minimizer $\phi[v]$ of the associated free energy $\mathcal{E}^{\delta, \operatorname{lin}}[v, \cdot]$ for which $2 \mathcal{W}^{\delta, \operatorname{lin}}[v, \phi[v]]=\mathcal{C}[\phi[v]]$. Hence, in this case we obtain $\overline{\mathcal{J}_{\mathcal{C}}^{\varepsilon}}=\mathcal{J}_{\mathcal{C}}^{\varepsilon}, \overline{\mathcal{J}_{\mathcal{C}}^{0}}=\underline{\mathcal{J}_{\mathcal{C}}^{0}}$ and thus the desired result applying Theorems 5.9 and 5.10 .

Finally, let us consider one particular compliance function. If we choose the dissipation associated with the transition from the unstressed state to the equilibrium deformation as compliance, $\mathcal{G}=\mathcal{D}$, instead of the internal elastic energy, $\mathcal{G}=\mathcal{W}$, or the change of external potential, $\mathcal{G}=\mathcal{C}$, we also obtain $\overline{\mathcal{J}_{\mathcal{D}}^{\varepsilon}}=\underline{\mathcal{J}_{\mathcal{D}}^{\varepsilon}}$ as well as $\overline{\mathcal{J}_{\mathcal{D}}^{0}}=\underline{\mathcal{J}_{\mathcal{D}}^{0}}$ by definition of $\mathcal{E}^{\delta}[v, \phi]$ and $\mathfrak{m}[v]$ (cf. also Rem. 5.8). Hence, we again obtain the following.

Corollary 5.13. Under the conditions of Theorem 5.4, we have

$$
\Gamma-\lim _{\varepsilon \rightarrow 0} \overline{\mathcal{J}_{\mathcal{D}}^{\varepsilon}}=\Gamma-\lim _{\varepsilon \rightarrow 0} \underline{\mathcal{J}_{\mathcal{D}}^{\varepsilon}}=\overline{\mathcal{J}_{\mathcal{D}}^{0}}=\underline{\mathcal{J}_{\mathcal{D}}^{0}}
$$

with respect to the $L^{1}(\Omega)$-topology.

\section{Numerical ALGORIthm}

In the following paragraphs, we will first state the optimality conditions of the minimization problem and its discretization by finite elements. We then briefly describe the computation of equilibrium deformations via a trust region method and the optimization for the phase field by a quasi-Newton method, embedded in a multiscale approach.

\subsection{Optimality conditions and finite element discretization}

A necessary condition for $\phi$ to satisfy the constraint of static equilibrium is that it fulfills the Euler-Lagrange condition

$$
0=\delta_{\phi} \mathcal{E}^{\delta}(\theta)=\int_{\Omega}\left((1-\delta) \chi_{\mathcal{O}}(v)+\delta\right) W_{, A}(\mathcal{D} \phi): \mathcal{D} \theta \mathrm{d} x-\int_{\Gamma_{N}} F \cdot \theta \mathrm{d} a
$$

for all test displacements $\theta: \Omega \rightarrow \mathbb{R}^{d}$ with $\left.\theta\right|_{\Gamma_{D}}=0$, where $\delta_{z} \mathcal{F}(\zeta)$ denotes the Gâteaux derivative of an energy $\mathcal{F}$ with respect to $z$ in some test direction $\zeta$. Hence, by the first order optimality conditions, the solution to our shape optimization problem can be described as a saddle point of the Lagrange functional

$$
L[v, \phi, p]=\mathcal{J}_{\mathcal{G}}^{\varepsilon}[v, \phi]+\delta_{\phi} \mathcal{E}^{\delta}[v, \phi](p)
$$

where $\mathcal{G}$ stands for $\mathcal{W}, \mathcal{C}$, or $\mathcal{D}, p$ denotes the Lagrange multiplier, and $\left.(\phi-\mathrm{id})\right|_{\Gamma_{D}}=\left.p\right|_{\Gamma_{D}}=0$. The associated necessary conditions are given by $0=\delta_{v} L=\delta_{\phi} L=\delta_{p} L$ with $\delta_{v} L=\delta_{v} \mathcal{R}+\nu \delta_{v} \mathcal{V}+\eta \delta_{v} \mathcal{L}^{\varepsilon}+\delta_{v} \delta_{\phi} \mathcal{E}^{\delta}(p)(\mathcal{R}$ here 
stands for $2 \mathcal{W}^{\delta}, \mathcal{C}$, or $\left.-2 \mathcal{E}^{\delta}\right), \delta_{\phi} L=\delta_{\phi} \mathcal{R}+\delta_{\phi} \delta_{\phi} \mathcal{E}^{\delta}(p), \delta_{p} L=\delta_{\phi} \mathcal{E}^{\delta}$, and

$$
\begin{aligned}
\delta_{v} \mathcal{V}(\vartheta) & =\int_{\Omega} \frac{\partial \chi_{\mathcal{O}}(v)}{\partial v} \vartheta \mathrm{d} x \\
\delta_{v} \mathcal{L}_{\mathrm{MM}}^{\varepsilon}(\vartheta) & =\int_{\Omega} \varepsilon \nabla v \cdot \nabla \vartheta+\frac{1}{2 \varepsilon} \frac{\partial \Psi(v)}{\partial v} \vartheta \mathrm{d} x \\
\delta_{v} \mathcal{W}^{\delta}(\vartheta) & =\int_{\Omega}(1-\delta) \frac{\partial \chi_{\mathcal{O}}(v)}{\partial v} \vartheta W(\mathcal{D} \phi) \mathrm{d} x \\
\delta_{\phi} \mathcal{W}^{\delta}(\theta) & =\int_{\Omega}\left((1-\delta) \chi_{\mathcal{O}}(v)+\delta\right) W_{, A}(\mathcal{D} \phi): \mathcal{D} \theta \mathrm{d} x \\
\delta_{\phi} \mathcal{C}(\theta) & =\int_{\Gamma_{N}} F \cdot \theta \mathrm{d} a \\
\delta_{v} \delta_{\phi} \mathcal{E}^{\delta}(p)(\vartheta) & =\int_{\Omega}(1-\delta) \frac{\partial \chi_{\mathcal{O}}(v)}{\partial v} \vartheta W_{, A}(\mathcal{D} \phi): \mathcal{D} p \mathrm{~d} x \\
\delta_{\phi} \delta_{\phi} \mathcal{E}^{\delta}(p)(\theta) & =\int_{\Omega}\left((1-\delta) \chi_{\mathcal{O}}(v)+\delta\right) W_{, A A}(\mathcal{D} \phi) \mathcal{D} \theta: \mathcal{D} p \mathrm{~d} x
\end{aligned}
$$

for scalar and vector-valued test functions $\vartheta$ and $\theta$, respectively, with $\left.\theta\right|_{\Gamma_{D}}=0$.

Furthermore, for a sufficiently smooth phase field $v$ and a deformation $\phi$ satisfying the equilibrium constraint, we may locally regard $\phi$ as a function $\phi[v]$. Then, by the adjoint method, the derivative of $\tilde{\mathcal{J}}_{\mathcal{G}}^{\varepsilon}[v]:=\mathcal{J}_{\mathcal{G}}^{\varepsilon}[v, \phi[v]]$ with respect to $v$ in direction $\vartheta$ is given as

$$
\delta_{v} \tilde{\mathcal{J}}_{\mathcal{G}}^{\varepsilon}(\vartheta)=\delta_{v} \mathcal{J}_{\mathcal{G}}^{\varepsilon}(\vartheta)+\delta_{v} \delta_{\phi} \mathcal{E}^{\delta}(p)(\vartheta)
$$

where for fixed $v$, the deformation $\phi[v]$ and the Lagrange multiplier $p$ solve $0=\delta_{\phi} L=\delta_{p} L$ with the corresponding Dirichlet boundary conditions at $\Gamma_{D}$. This directional derivative can be used in gradient descent type algorithms to find the optimal phase field $v$.

Concerning the discretization, we restrict ourselves to problems in two dimensions $(d=2)$ and approximate the phase field $v$ and deformation $\phi$ by continuous, piecewise multilinear finite element functions $V$ and $\Phi$ on a regular mesh on $\Omega=[0,1]^{2}$ with $2^{L}+1$ nodes in each space direction. The different energy terms $\mathcal{W}^{\delta}, \mathcal{V}$, and $\mathcal{L}_{\mathrm{MM}}^{\varepsilon}$ are approximated by third-order Gaussian quadrature on each grid cell. For the ease of presentation, in what follows we implicitly assume that the evaluation of every functional on finite element input functions is performed only approximately using this quadrature. In our applications, for the sake of simplicity we restrict both $\Gamma_{D}$ and $\Gamma_{N}$ to a union of several grid cell faces so that in particular $\Gamma_{N}$ is discretized in the canonical way by a regular mesh on which a continuous, piecewise multilinear finite element approximation of the surface load $F$ can be defined. $\mathcal{C}$ is then also computed on this lower dimensional finite element mesh.

\subsection{Inner minimization to find equilibrium deformation}

We aim at a gradient descent type algorithm for the (discretized) phase field $V$, where in each step we first minimize $\mathcal{E}^{\delta}[V, \Phi]$ to obtain a finite-element approximation $\Phi[V]$ of the equilibrium deformation and then use this deformation to evaluate the compliance functionals $\mathcal{J}_{\mathcal{W}}^{\varepsilon}[V, \Phi[V]]$ or $\mathcal{J}_{\mathcal{C}}^{\varepsilon}[V, \Phi[V]]$ and their Gâteaux derivative with respect to $V$. The inner minimization of $\mathcal{E}^{\delta}[V, \Phi]$ for $\Phi$ has to meet particularly strong requirements. First of all, the optimal deformation $\Phi[V]$ has to be accurately found in order to enable a correct evaluation of the objective energy and to obtain a good approximation of the Gâteaux derivative which can then be used to compute a descent direction. Second, since the minimization has to be performed for each energy evaluation, we need a fast converging method. Finally, the optimization method has to be very robust and should reliably lead to a (local) minimum.

The robustness requirement is particularly related to the use of the nonlinear elastic energy: In the presence of buckling instabilities, there is typically an unstable or metastable (meaning that small perturbations suffice 

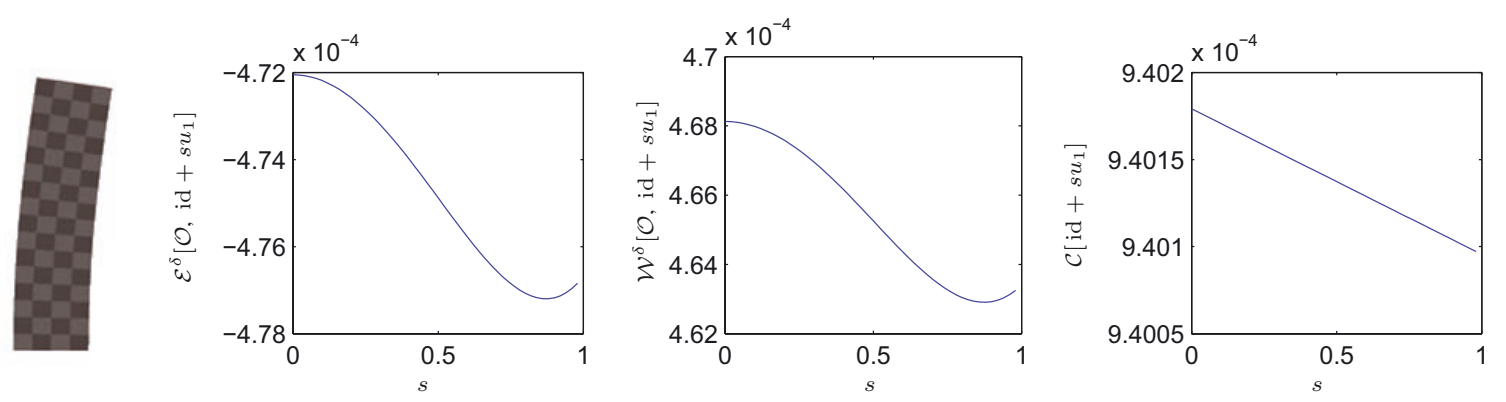

FIGURE 10. Left: Eigendisplacement of a symmetrically compressed beam (height $L=1$, thickness $t=0.25$, Young's modulus $E=4$ ) corresponding to the negative eigenvalue of the free energy Hessian. Right: Energetic changes for the perturbation of the symmetric compression in direction of the eigendisplacement. The coordinate $s$ indicates the perturbation strength, and $s=0$ corresponds to the symmetric deformation.

to abandon the state), non-buckled state of the deformation $\Phi$ which more or less corresponds to the deformation in the linearized elastic setting. This state is associated with a saddle point of the energy $\mathcal{E}^{\delta}[V, \Phi]$, which has to be robustly bypassed by the minimization method. While simple gradient descent type methods tend to slow down considerably in the vicinity of such points, the basic Newton algorithm is prone to converge exactly to this saddle point. Indeed, recalling the simulations of buckling rods from Figure 5, for symmetry reasons we know that the symmetric deformation in between buckling deformations to both sides must be a critical point of $\mathcal{E}^{\delta}[\mathcal{O}, \cdot]$. This deformation is readily obtained by a simple Newton iteration to find the zero of the derivative of $\mathcal{E}^{\delta}[\mathcal{O}, \cdot]$. The corresponding stiffness operator, that is, the Hessian of $\mathcal{E}^{\delta}[\mathcal{O}, \cdot]$ at this symmetric deformation then is indeed indefinite and has a negative eigenvalue, classifying the symmetric deformation as a saddle point of the free energy. For the bar of height $L=1$, thickness $t=0.25$, and Young's modulus $E=4$ the eigendisplacement $u_{1}$ belonging to the negative eigenvalue $\lambda_{1}=-2.7 \cdot 10^{-5}$ is shown in Figure 10 , as well as the decrease of $\mathcal{E}^{\delta}\left[\mathcal{O}\right.$, id $\left.+s u_{1}\right], \mathcal{W}^{\delta}\left[\mathcal{O}\right.$, id $\left.+s u_{1}\right]$, and $\mathcal{C}\left[\mathrm{id}+s u_{1}\right]$ along this direction for increasing values of $s$. In fact, the eigendisplacement can easily be recognized as a (linearized) bending deformation.

Due to the above-mentioned problems of simple gradient-descent or Newton methods, we will need a more sophisticated technique. Furthermore, the energy landscape in the nonlinear regime is typically characterized by long, deep, narrow and bent valleys. These valleys may be interpreted as the paths along which the material can be deformed, and leaving these valleys will rapidly lead to unphysical states such as local material interpenetration and thus the break-down of the minimization.

Trust region methods represent a very reliable minimization technique that satisfies all the above issues. At each step $i$, the objective functional is approximated by a quadratic model $m_{i}$ which is minimized inside a so-called trust region around $\Phi_{i}$ to obtain a new guess $\Phi_{i+1}$. If the decrease of the objective functional agrees sufficiently with the decrease of the quadratic model, the step is accepted and the trust region enlarged; otherwise, the trust region is shrunken.

The subtleties of a trust-region method lie in the treatment of the so-called trust region subproblem to minimize the quadratic model within the trust region. In our computations, we chose to implement the algorithm proposed in [19], Algorithm 7.3.4. At step $i$, the quadratic model $m_{i}$ is given as the second order Taylor expansion of the discrete energy $\mathcal{E}^{\delta}[V, \cdot]$ about $\Phi_{i}$, which involves the Hessian $H\left(\Phi_{i}\right)$ of $\mathcal{E}^{\delta}[V, \cdot]$. To minimize this model within a circular trust region around $\Phi_{i}$ of radius $\Delta$, the smallest positive scalar $\xi$ is sought such that $H_{i}(\xi):=H\left(\Phi_{i}\right)+\xi$ id becomes positive definite and the global minimum of the correspondingly modified Taylor expansions lies within the trust region. The positive definiteness of the quadratic operator is checked via a Cholesky factorization $H_{i}(\xi)=L L^{\mathrm{T}}$, which also serves to find the minimum by solving the corresponding linear system of equations that results from the optimality conditions. Additionally, the eigendirection belonging to the smallest eigenvalue of $H_{i}(\xi)$ is approximated by a technique which aims to find a vector $Y$ such that $L^{-1} Y$ 
is large. This eigendirection is essentially employed to bypass saddle points. The scalar $\xi$ is itself obtained by a Newton iteration which is safeguarded by a number of sophisticated bounds on $\xi$ (see [19] for details). The Cholesky factorization is performed using the CHOLMOD package from Davis et al. [17,22], where a matrix reordering ensures a minimum fill-in.

In our setting, the discrete energy gradient and the Hessian matrix $H(\Phi)$ are evaluated as

$$
\left(\delta_{\phi} \mathcal{E}^{\delta}\left(\varphi_{i} e_{j}\right)\right)_{(i, j) \in I_{h}^{0} \times\{1,2\}}=\left(\int_{\Omega}\left((1-\delta) \chi_{\mathcal{O}}(V)+\delta\right) W_{, A}(\mathcal{D} \Phi): \mathcal{D}\left(\varphi_{i} e_{j}\right) \mathrm{d} x-\int_{\Gamma_{N}} F \cdot\left(\varphi_{i} e_{j}\right) \mathrm{d} a\right)_{(i, j) \in I_{h}^{0} \times\{1,2\}}
$$

and

$$
\begin{aligned}
H & =\left(\delta_{\phi \phi} \mathcal{E}^{\delta}\left(\varphi_{i} e_{j}, \varphi_{k} e_{l}\right)\right)_{(i, j),(k, l) \in I_{h}^{0} \times\{1,2\}} \\
& =\left(\int_{\Omega}\left((1-\delta) \chi_{\mathcal{O}}(V)+\delta\right) W_{, A A}(\mathcal{D} \Phi) \mathcal{D}\left(\varphi_{i} e_{j}\right): \mathcal{D}\left(\varphi_{k} e_{l}\right) \mathrm{d} x\right)_{(i, j),(k, l) \in I_{h}^{0} \times\{1,2\}}
\end{aligned}
$$

for the set $I_{h}^{0}$ of node indices in $\Omega \backslash \Gamma_{D}$, the finite element basis functions $\left\{\varphi_{i}\right\}_{i \in I_{h}^{0}}$, and the canonical Euclidean basis $\left\{e_{1}, e_{2}\right\}$ in $\mathbb{R}^{2}$.

\subsection{Optimization for the phase field}

Concerning the outer optimization for $V$, we apply a Davidon-Fletcher-Powell quasi-Newton method, which - expressed for the minimization of a function $f: \mathbb{R}^{N} \rightarrow \mathbb{R}, x \mapsto f(x)$ - uses the update formula

$$
B_{k+1}=B_{k}+\frac{\Delta x_{k} \Delta x_{k}^{T}}{g_{k}^{T} \Delta x_{k}}-\frac{B_{k} g_{k} g_{k}^{T} B_{k}^{T}}{g_{k}^{T} B_{k} g_{k}}
$$

to approximate the inverse of the Hessian of $f$ in the $(k+1)$ th step using the latest update $\Delta x_{k}=x_{k+1}-x_{k}$ and the difference $g_{k}=\nabla f\left(x_{k+1}\right)-\nabla f\left(x_{k}\right)$ between the gradients. The descent direction $p_{k}$ is then chosen as $-B_{k} \nabla f\left(x_{k}\right)$, and the step length $\tau_{k}$ is determined to satisfy the strong Wolfe conditions,

$$
\begin{aligned}
f\left(x_{k}+\tau_{k} p_{k}\right) & \leq f\left(x_{k}\right)+c_{1} \tau_{k} \nabla f_{k} \cdot p_{k}, \\
\left|\nabla f\left(x_{k}+\tau_{k} p_{k}\right) \cdot p_{k}\right| & \leq c_{2}\left|\nabla f\left(x_{k}\right) \cdot p_{k}\right|
\end{aligned}
$$

for $c_{1}=0.5, c_{2}=0.9$. Furthermore, we reset $B_{k}$ to the identity every tenth step to restrict memory usage and to ensure a descent at least as good as gradient descent.

The gradient of the objective functional $\tilde{\mathcal{J}}_{\mathcal{G}}^{\varepsilon}[V]=\mathcal{J}_{\mathcal{G}}^{\varepsilon}[V, \Phi[V]]$ (for $\mathcal{G}=\mathcal{W}$ or $\mathcal{G}=\mathcal{C}$ ) with respect to $V$ is computed via the adjoint method as described in Section 6.1. We first solve

$$
\delta_{\phi} \delta_{\phi} \mathcal{E}^{\delta}(\Psi)(P)=-\delta_{\phi} \mathcal{J}_{\mathcal{G}}^{\varepsilon}(\Psi)
$$

for the finite-element Lagrange multiplier $P$ under the constraint $\left.P\right|_{\Gamma_{D}}=0$, where $\Psi$ runs over all vector-valued finite-element functions that are zero on $\Gamma_{D}$. In terms of finite-element operators, this can be expressed as the linear system $H \mathbf{P}=\mathbf{R}$, where $\mathbf{P}$ denotes the vector of nodal values of $P$ on $\Omega \backslash \Gamma_{D}$, the matrix $H$ has been given above, and the right-hand side reads

$$
\begin{aligned}
\mathbf{R} & =\left(\int_{\Omega}\left((1-\delta) \chi_{\mathcal{O}}(V)+\delta\right) W_{, A}(\mathcal{D} \Phi[V]): \mathcal{D}\left(\varphi_{i} e_{j}\right) \mathrm{d} x\right)_{(i, j) \in I_{h}^{0} \times\{1,2\}} \\
\text { or } \quad \mathbf{R} & =\left(\int_{\Gamma_{N}} F \cdot\left(\varphi_{i} e_{j}\right) \mathrm{d} a\right)_{(i, j) \in I_{h}^{0} \times\{1,2\}},
\end{aligned}
$$


depending on whether $\mathcal{J}_{\mathcal{W}}^{\varepsilon}[V, \Phi[V]]$ or $\mathcal{J}_{\mathcal{C}}^{\varepsilon}[V, \Phi[V]]$ is minimized. Then we obtain the gradient of the objective functional with respect to $V$ as

$$
\left(\delta_{v} \mathcal{J}_{\mathcal{G}}^{\mathcal{E}}\left(\varphi_{i}\right)+\delta_{v} \delta_{\phi} \mathcal{E}^{\delta}(P)\left(\varphi_{i}\right)\right)_{i \in I_{h}}
$$

for all $i \in I_{h}$, where $I_{h}$ represents the set of all node indices in $\Omega$ except those at which $V$ is fixed by a Dirichlet condition, and where the expressions for the Gâteaux derivatives are provided in Section 6.1.

\subsection{Embedding the optimization in a multiscale approach}

In order to enhance convergence and to avoid local minima, we pursue a multiscale approach, using a hierarchy of dyadic grid resolutions with $2^{l}+1$ nodes in each direction and multilinear interpolation as prolongation technique. We first perform the minimization for a coarse spatial discretization and then successively prolongate and refine the result on finer grids. The phase-field scale parameter $\varepsilon$ is coupled to the grid size $h$ via $\varepsilon=h$ in order to allow a sufficient resolution of the interface. Finally, it is sometimes advantageous to take a smaller value for $\nu$ on coarse grids in order not to penalize the value $V=1$ so strongly that intermediate values of $V$ between -1 and 1 are preferred. As the grid gets finer, $\nu$ can be increased since the smaller value of $\varepsilon$ forces the phase-field values towards the pure phases -1 and 1 .

The multiscale approach has the advantage that coarse structures already develop on a coarse scale so that the finer scales only have to recover the details. If these coarse structures have to be found directly on the finest level, much more quasi-Newton iterations are needed since the interfaces propagate much more slowly. In general, quite few iterations on each coarse level already suffice to roughly position all coarse structures (typically far less than 100 steps in our experiments). The iteration on the finest level then needs up to several hundred steps. In contrast, an iteration directly started on the finest grid needs more than ten times as many steps (compare Fig. 15).

A brief overview over the entire algorithm in pseudo code notation reads as follows (bold capital letters represent vectors of nodal values, and $\mathcal{G}$ stands for $\mathcal{W}, \mathcal{C}$, or $\mathcal{D}$ ):

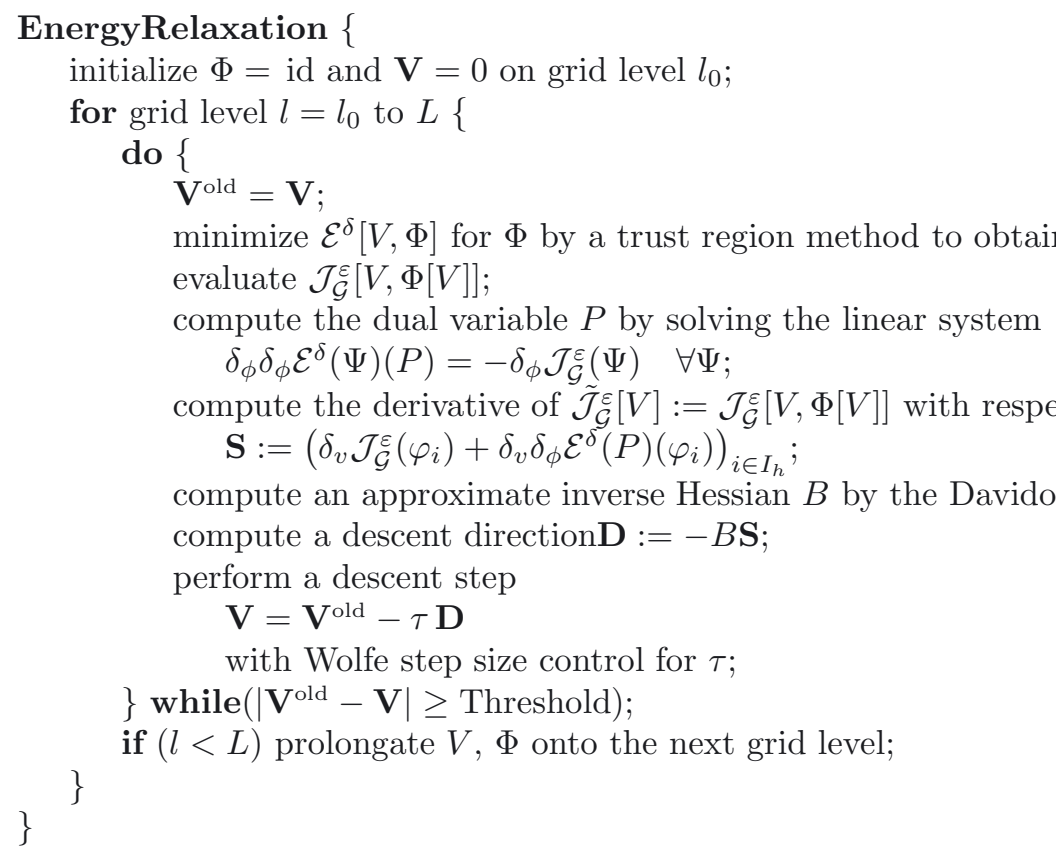




\section{EXPERIMENTS}

The effect of using nonlinear instead of linearized elasticity has already been explored in Figure 3, where we compared optimal cantilever shapes for loads of different magnitudes, using $\mathcal{J}_{\mathcal{C}}^{\varepsilon}$ as objective functional. White and black regions correspond to the phases $v=1$ and $v=-1$, respectively. Of course, increasing the load results in stronger deformations and thus higher values of $\mathcal{C}$ (and $\mathcal{W}$ ) so that the shapes would naturally become thicker and more strutted in order to balance the compliance with the volume costs $\mathcal{V}$ and the perimeter regularization $\mathcal{L}_{\mathrm{MM}}^{\varepsilon}$. To make the optimal designs comparable and to reveal the pure influence of introducing geometric and material nonlinearity, the weights $\nu$ and $\eta$ of $\mathcal{V}$ and $\mathcal{L}_{\mathrm{MM}}^{\varepsilon}$ have to be increased in parallel. Since the compliance scales quadratically with the load (at least for small deformations in the regime of linearized elasticity), $\nu$ and $\eta$ are chosen such that $\frac{F^{2}}{\nu}$ and $\frac{F^{2}}{\eta}$ stay constant.

As discussed in Section 4, compared to optimal designs for linearized elasticity, the symmetry of the cantilever design is broken due to the nonlinear influence of the loading direction. We observe that as the load increases, a structure evolves which exhibits a single thick beam at the top that is supported from below by several thinner struts extending from the wall to the point where the load is applied as well as to two or three other points along the beam. These struts are themselves suspended from thread-like structures.

In the beginning, we have stated and discussed the different possibilities to extend the notion of compliance to the setting of nonlinear elasticity. In particular we have considered the change of external potential $\mathcal{C}$ and the internally stored elastic energy $\mathcal{W}$. The compliance minimization yields different results depending on our choice: While the use of $\mathcal{C}$ will produce rather rigid constructions that allow only small displacements, the use of $\mathcal{W}$ does in principle allow for large deformations as long as the final equilibrium state is not heavily strained. As a third possibility we have introduced the energy dissipation $-2 \mathcal{E}=2 \mathcal{C}-2 \mathcal{W}$.

A comparison of the three possibilities reveals a complex interplay between the volume costs and the mechanical energy (Fig. 11). We first observe that the obtained shapes are plausible in the sense that each of them indeed has the minimum value of its associated objective function $\left(\mathcal{J}_{\mathcal{C}}^{\varepsilon}, \mathcal{J}_{\mathcal{W}}^{\varepsilon}\right.$, or $\mathcal{J}_{\mathcal{D}}^{\varepsilon}$, respectively) among all three. However, we also notice that the various energy contributions do not differ significantly between the three designs. Nevertheless, they do look quite different: While the minimizer of $\mathcal{J}_{\mathcal{D}}$ is almost symmetric, the minimizer of $\mathcal{J}_{\mathcal{W}}$ is strongly asymmetric. In particular, the bottommost beam, which will be compressed during the deformation, becomes thinner from $\mathcal{J}_{\mathcal{D}}$ to $\mathcal{J}_{\mathcal{C}}$ to $\mathcal{J}_{\mathcal{W}}$, thereby allowing stronger displacements. This agrees with the simplified model example in Section 3. The internal energy $\mathcal{W}$ seems to be not very sensitive to the thickness of the bottommost beam, while the potential change $\mathcal{C}$ strongly is. Therefore, it pays off to save volume at the cost of a slightly increasing $\mathcal{W}$ in order to minimize $\mathcal{J}_{\mathcal{W}}$. In fact, the model example in Section 3 already suggests that the middle design in Figure 11 is only locally optimal. Indeed, if we initialize the shape as a simple rod similar to Figure 2, then we obtain the design shown in Figure 12 with a much lower objective function value.

Apart from the employed hyperelastic material law, there are two weighting parameters that need to be tuned in order to obtain sensible results. Consequently, we have to study the influence of the different energy contributions. The impact of volume penalization and perimeter regularization seems clear; the former prefers thinner structures while the latter tends to reduce the degree of cross-linking. The question arises how the compliance term affects the optimal shape, whether by thickening its single components or by producing more strutted shapes. Experiments show that both mechanisms occur until a point at which the different material parts have grown up to a width that they start to merge and eliminate any fine structure (Fig. 13).

To explore the effect of varying the two weight parameters $\nu$ and $\eta$ systematically, let us sample the twoparameter family of (at least locally) optimal shapes which is generated by $\eta$ and $\nu$. Before doing so, note that generally, the maximally reachable resolution of our optimal designs is restricted due to computation time, which in turn also fixes the smallest resolvable scale parameter $\varepsilon$. Since the phase field $v$ is only forced towards the pure phases $v \in\{-1,1\}$ for the limit $\varepsilon \rightarrow 0$, there will inherently always remain intermediate values (which can also be observed in some of the previous examples). One particular reason for this lies in the strong interaction between the non-convex potential $\eta \frac{9}{32} \frac{1}{\varepsilon}\left(v^{2}-1\right)^{2}$ with the two energy-minimizing phases $v \in\{-1,1\}$ 


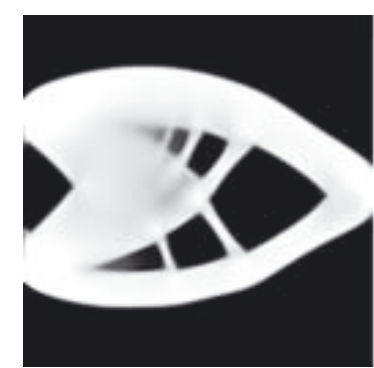

$$
\begin{aligned}
\mathcal{L}_{\mathrm{MM}}^{\varepsilon}[u] & =9.9525 \\
\mathcal{V}[u] & =0.3590 \\
\mathcal{C}[\phi[u]] & =0.6687 \\
\mathcal{W}^{\delta}[v, \phi[u]] & =0.3308 \\
\mathcal{E}[u, \phi[u]] & =-0.3380 \\
\mathcal{J}_{\mathcal{C}}^{\varepsilon}[u, \phi[u]] & =1.3078 \\
\mathcal{J}_{\mathcal{W}}^{\varepsilon}[u, \phi[u]] & =1.3007 \\
\mathcal{J}_{\mathcal{D}}^{\varepsilon}[u, \phi[u]] & =1.3150
\end{aligned}
$$

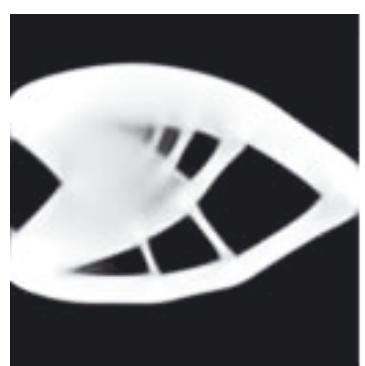

$$
\mathcal{L}_{\mathrm{MM}}^{\varepsilon}[u]=10.0343
$$$$
\mathcal{V}[u]=0.3481
$$$$
\mathcal{C}[\phi[u]]=0.6896
$$$$
\mathcal{W}^{\delta}[v, \phi[u]]=0.3383
$$$$
\mathcal{E}[u, \phi[u]]=-0.3514
$$$$
\mathcal{J}_{\mathcal{C}}^{\mathcal{E}}[u, \phi[u]]=1.3113
$$$$
\mathcal{J}_{\mathcal{W}}^{\varepsilon}[u, \phi[u]]=1.2982
$$$$
\mathcal{J}_{\mathcal{D}}^{\varepsilon}[u, \phi[u]]=1.3244
$$

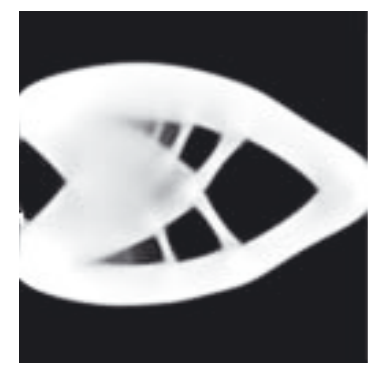

$$
\begin{aligned}
\mathcal{L}_{\mathrm{MM}}^{\varepsilon}[u] & =10.0863 \\
\mathcal{V}[u] & =0.3648 \\
\mathcal{C}[\phi[u]] & =0.6599 \\
\mathcal{W}^{\delta}[v, \phi[u]] & =0.3284 \\
\mathcal{E}[u, \phi[u]] & =-0.3314 \\
\mathcal{J}_{\mathcal{C}}^{\varepsilon}[u, \phi[u]] & =1.3092 \\
\mathcal{J}_{\mathcal{W}}^{\varepsilon}[u, \phi[u]] & =1.3062 \\
\mathcal{J}_{\mathcal{D}}^{\varepsilon}[u, \phi[u]] & =1.3122
\end{aligned}
$$

Figure 11. Optimal cantilever design for minimizing $\mathcal{J}_{\mathcal{C}}^{\varepsilon}, \mathcal{J}_{\mathcal{W}}^{\varepsilon}, \mathcal{J}_{\mathcal{D}}^{\varepsilon}$ (from left to right), taking the same parameters as in Figure 3 for the case $\hat{F}=4$ (in these computations, $\varepsilon$ was chosen half the grid size $h$ in order to obtain acceptable phase fields already for a resolution of $129 \times 129)$. Minimization of $\mathcal{J}_{\mathcal{D}}^{\varepsilon}$ (right) yields the most symmetric shape, while minimization of $\mathcal{J}_{\mathcal{W}}^{\varepsilon}$ (middle) yields the most asymmetric one with the compressed struts being significantly thinner than in the other cases.

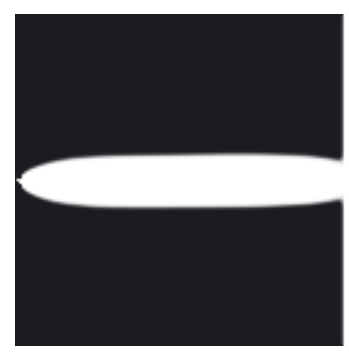

$$
\begin{aligned}
\mathcal{L}_{\mathrm{MM}}^{\mathcal{E}}[u] & =3.9006 \\
\mathcal{V}[u] & =0.1385 \\
\mathcal{C}[\phi[u]] & =4.3841 \\
\mathcal{W}^{\delta}[v, \phi[u]] & =0.2077 \\
\mathcal{E}[u, \phi[u]] & =-4.1764 \\
\mathcal{J}_{\mathcal{C}}^{\mathcal{E}}[u, \phi[u]] & =4.6310 \\
\mathcal{J}_{\mathcal{W}}^{\varepsilon}[u, \phi[u]] & =0.6623 \\
\mathcal{J}_{-\mathcal{E}}^{\mathcal{E}}[u, \phi[u]] & =8.5997
\end{aligned}
$$

FIGURE 12. Initializing the phase field as a single rod, a minimization of $\mathcal{J}_{\mathcal{W}}^{\varepsilon}$ retrieves the above optimal cantilever design with a much lower objective function value than the design in Figure 11, middle.

and the convex volume $\operatorname{costs} \frac{\nu}{4}(v+1)^{2}$. For too large $\varepsilon$, that is, for $\varepsilon \geq \frac{\eta}{\nu} \frac{9}{4}$, their sum becomes convex so that there are no longer two preferred phases. Due to the significance of $\frac{\nu}{\eta}$ in this lower bound, in our parameter study we shall choose to vary $\frac{\nu}{\eta}$ and $\eta$ instead of $\nu$ and $\eta$.

Figure 14 shows cantilever designs which were obtained by choosing the middle computation as the reference setting and then doubling or halving $\frac{\nu}{\eta}$ as well as $\eta$. Apparently, for fixed $\varepsilon$ there is a regime of $\frac{\nu}{\eta}$ for which reasonable shapes are obtained, corresponding to the middle column. For larger values, phase-field densities 

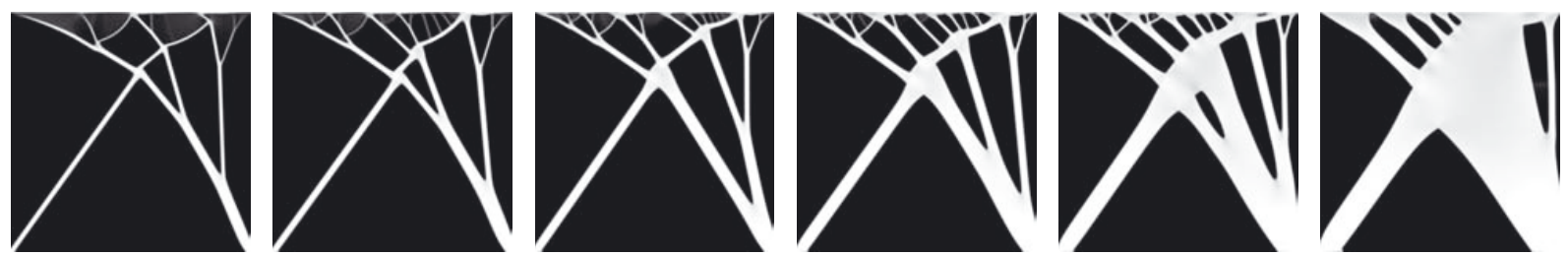

Figure 13. Optimal designs with respect to $\mathcal{J}_{\mathcal{C}}^{\varepsilon}$ for the same load case as in Figure 6 with parameters $F=0.016 \cdot \sqrt{2}, \lambda=\mu=80, \eta=2^{k} \cdot 10^{-8}, \nu=2^{5+k} \cdot 10^{-7}$ for $k=6, \ldots, 1$ from left to right (resolution $512 \times 512$ ). The load is chosen very small so as to stay in the regime of linearized elasticity such that buckling cannot occur for the fine shapes on the left.

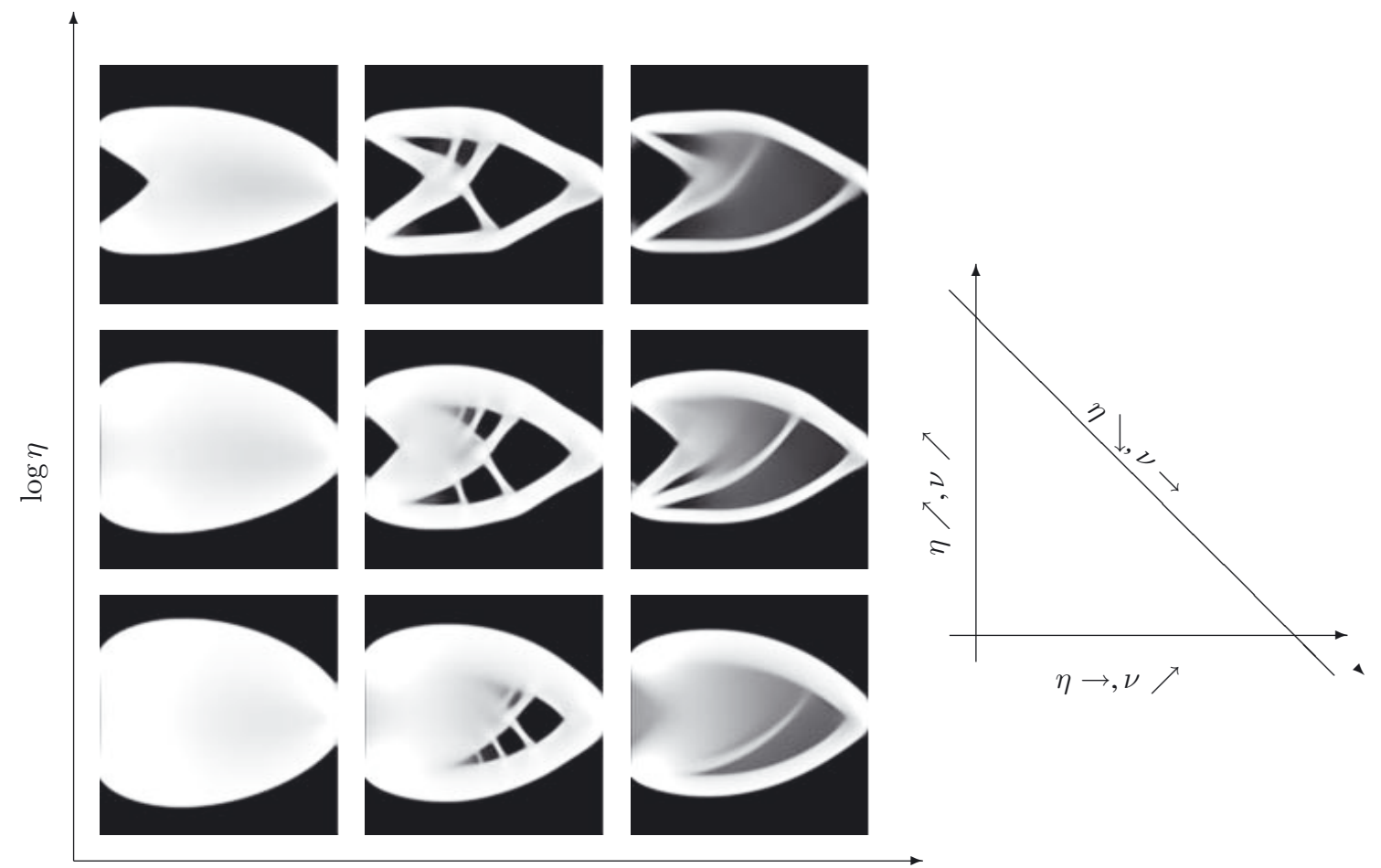

Figure 14. Optimal cantilever designs for different parameter values. The middle design is the same as in Figure 11, left; the top and bottom row as well as the right and left column are obtained by doubling and halving $\eta$ and $\frac{\nu}{\eta}$, respectively.

between -1 and 1 are not sufficiently penalized, and for smaller values we obtain bulky designs without any fine structure. The effect of varying $\eta$ for a constant volume weight $\nu$ can be seen along the descending diagonals; these designs indeed seem to possess similar volumes, but a distinct amount of fine structure. Along the horizontal direction, just $\nu$ changes, and the design volumes can be seen to decrease to the right.

Naturally, the energy landscape associated with $\mathcal{J}_{\mathcal{C}}^{\varepsilon}, \mathcal{J}_{\mathcal{W}}^{\varepsilon}$, and $\mathcal{J}_{\mathcal{D}}^{\varepsilon}$ is quite complicated and allows for multiple local minima. In order to reduce the influence of initialization and to obtain satisfactory results with a sufficiently low objective function value, in the above examples we pursued a multiscale approach with an initial optimization on a coarse resolution (to find a good large scale structure) and successive refinement. For initialization on the coarsest level, each nodal value of the phase field is taken randomly from a uniform distribution on $[-0.1,0.1]$. Also, we started with a small value of $\nu$ on the coarse grid and then doubled it at each prolongation until 


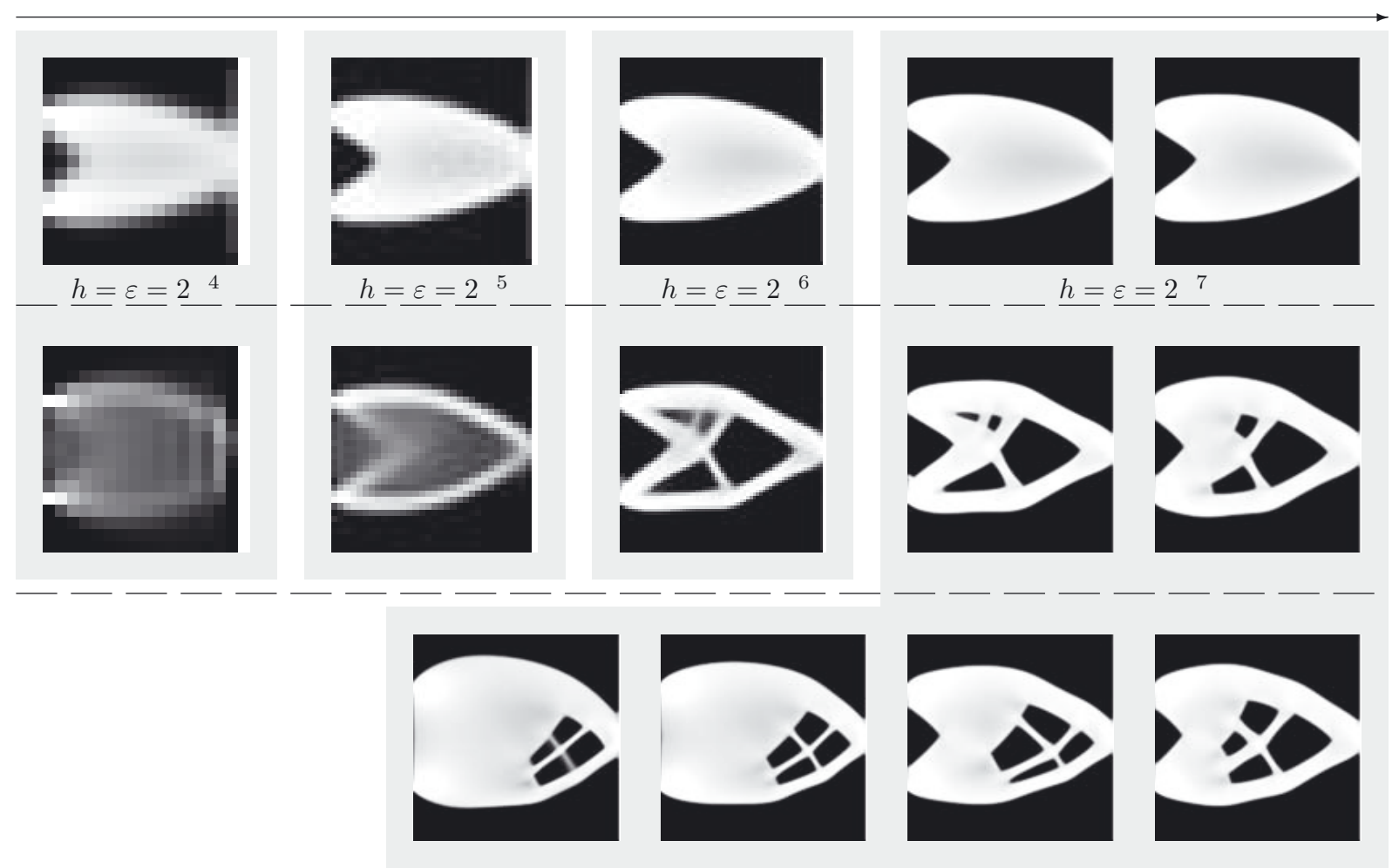

FIGURE 15. Intermediate results of the optimization for the top left case of Figure 14. The first three images in the top row show the results of the multiscale algorithm on grid levels four to six, where $\nu$ has been doubled at each prolongation. The rightmost two images show the shape after 300 quasi-Newton iterations on grid level seven as well as the final result. The middle row shows the same results, only that this time $\nu$ is the same on all grid levels. In the bottom row, the optimization was started directly on grid level seven without a multiscale approach. The intermediate shapes after 300, 600, and 3000 iterations as well as the final result are depicted. The final values of the objective function $\mathcal{J}_{\mathcal{C}}^{\varepsilon}$ are $1.3500,1.3229,1.3239$, respectively.

its final value on the finest grid level. In this way we maintain a constant ratio $\frac{\eta}{\varepsilon \nu}$ over all grid levels so that volume costs and chemical potential already balance each other on the coarser grid levels and the coarse phase fields are already quite close to an optimal design (compare Fig. 15).

Alternatively, one could employ the same value of $\nu$ on all grid levels or start the optimization directly on the finest level without a multiscale approach. While all three approaches yield the same result for many parameter constellations (which is not too surprising especially for the rightmost column in Fig. 14, for example, where we have reached the regime in which the sum of volume costs and chemical potential are convex), this does not hold for some cases as for example depicted in Figure 15, where the progress of the optimization algorithm is shown. In general, however, the achieved objective function values are very close to each other, and in some cases it seems that at least two of the tree approaches would eventually converge against the same shape if they were not terminated due to too small progress. Note that for the case in Figure 15, one approach seems not to be able to create holes inside the bulky shape.

As a final example, we have computed an optimal design for a bridge-like structure with two pointwise Dirichlet boundary conditions and a uniform downward surface load (Fig. 16). Apparently, the optimal design is to suspend the bottom edge from an arch extending between both fixing points. In order to reduce computation 

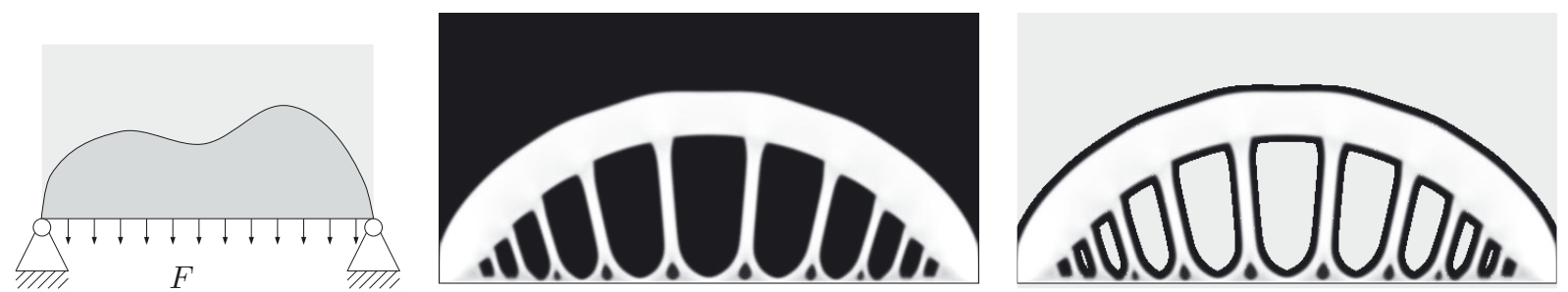

Figure 16. Sketch of the design problem and optimal design for $F=1.2, \lambda=\mu=80$, $\eta=10^{-4}, \nu=2^{5} \cdot 10^{-3}$. The right image shows the final, actual computational domain: The light gray background does not belong to the computational domain whereas the foreground (which is cut out of the middle image) does.

time for this example (especially during the Cholesky-factorization), we did not use the entire unit square $[0,1]^{2}$ as the computational domain, but instead updated the computational domain every 100 iterations as the region $\left\{x \in[0,1]^{2}: v(x)>-0.95\right\}$, dilated by three grid cells. This update does not hamper convergence, and the final, actual computational domain is shown in Figure 16, right.

Acknowledgements. Benedikt Wirth has been funded by the Bonn International Graduate School in Mathematics. The work was supported by the Deutsche Forschungsgemeinschaft, SPP 1253 (Optimization with Partial Differential Equations).

\section{REFERENCES}

[1] G. Allaire, Shape optimization by the homogenization method, Applied Mathematical Sciences 146. Springer-Verlag, New York (2002).

[2] G. Allaire, Topology Optimization with the Homogenization and the Level-Set Method, in Nonlinear Homogenization and its Applications to Composites, Polycrystals and Smart Materials, NATO Science Series II: Mathematics, Physics and Chemistry 170, Springer (2004) 1-13.

[3] G. Allaire, E. Bonnetier, G. Francfort and F. Jouve, Shape optimization by the homogenization method. Numer. Math. 76 (1997) 27-68.

[4] G. Allaire, F. Jouve and A.-M. Toader, A level-set method for shape optimization. C. R. Acad. Sci. Paris, Sér. I 334 (2002) $1125-1130$.

[5] G. Allaire, F. Jouve and H. Maillot, Topology optimization for minimum stress design with the homogenization method. Struct. Multidisc. Optim. 28 (2004) 87-98.

[6] G. Allaire, F. Jouve and A.-M. Toader, Structural optimization using sensitivity analysis and a level-set method. J. Comput. Phys. 194 (2004) 363-393.

[7] G. Allaire, F. de Gournay, F. Jouve and A.-M. Toader, Structural optimization using topological and shape sensitivity via a level set method. Control Cybern. 34 (2005) 59-80.

[8] S.M. Allen and J.W. Cahn, A microscopic theory for antiphase boundary motion and its application to antiphase domain coarsening. Acta Metall. 27 (1979) 1085-1095.

[9] L. Ambrosio and G. Buttazzo, An optimal design problem with perimeter penalization. Calc. Var. 1 (1993) 55-69.

[10] R. Ansola, E. Veguería, J. Canales and J.A. Tárrago, A simple evolutionary topology optimization procedure for compliant mechanism design. Finite Elements Anal. Des. 44 (2007) 53-62.

[11] J.M. Ball, Convexity conditions and existence theorems in nonlinear elasiticity. Arch. Ration. Mech. Anal. 63 (1977) $337-403$.

[12] J.M. Ball, Global invertibility of Sobolev functions and the interpenetration of matter. Proc. R. Soc. Edinb. A 88 (1981) 315-328.

[13] B. Bourdin and A. Chambolle, Design-dependent loads in topology optimization. ESAIM: COCV 9 (2003) 19-48.

[14] A. Braides, $\Gamma$-convergence for beginners, Oxford Lecture Series in Mathematics and its Applications 2. Oxford University Press, Oxford (2002).

[15] M. Burger and R. Stainko, Phase-field relaxation of topology optimization with local stress constraints. SIAM J. Control Optim. 45 (2006) 1447-1466.

[16] A. Chambolle, A density result in two-dimensional linearized elasticity, and applications. Arch. Ration. Mech. Anal. 167 (2003) 211-233. 
[17] Y. Chen, T.A. Davis, W.W. Hager and S. Rajamanickam, Algorithm 887: CHOLMOD, supernodal sparse Cholesky factorization and update/downdate. ACM Trans. Math. Softw. 35 (2009) 22:1-22:14.

[18] P.G. Ciarlet, Three-dimensional elasticity. Elsevier Science Publishers B. V. (1988).

[19] A.R. Conn, N.I.M Gould and P.L. Toint, Trust-Region Methods. SIAM (2000).

[20] S. Conti, H. Held, M. Pach, M. Rumpf and R. Schultz, Risk averse shape optimization. SIAM J. Control Optim. (to appear).

[21] B. Dacorogna, Direct methods in the calculus of variations. Springer-Verlag, New York (1989).

[22] T.A. Davis and W.W. Hager, Dynamic supernodes in sparse Cholesky update/downdate and triangular solves. ACM Trans. Math. Softw. 35 (2009) 27:1-27:23.

[23] G.P. Dias, J. Herskovits and F.A. Rochinha, Simultaneous shape optimization and nonlinear analysis of elastic solids, in Computational Mechanics - New Trends and Applications, E. Onate, I. Idelsohn and E. Dvorkin Eds., CIMNE, Barcelona (1998) 1-13.

[24] X. Guo, K. Zhao and M.Y. Wang, Simultaneous shape and topology optimization with implicit topology description functions. Control Cybern. 34 (2005) 255-282.

[25] Z. Liu, J.G. Korvink and R. Huang, Structure topology optimization: Fully coupled level set method via femlab. Struct. Multidisc. Optim. 29 (2005) 407-417.

[26] J.E. Marsden and T.J.R. Hughes, Mathematical Foundations of Elasticity. Prentice-Hall, Englewood Cliffs (1983).

[27] L. Modica and S. Mortola, Un esempio di $\Gamma^{-}$-convergenza. Boll. Un. Mat. Ital. B (5) 14 (1977) 285-299.

[28] P. Pedregal, Variational Methods in Nonlinear Elasticity. SIAM (2000).

[29] J.A. Sethian and A. Wiegmann, Structural boundary design via level set and immersed interface methods. J. Comput. Phys. 163 (2000) 489-528.

[30] O. Sigmund and P.M. Clausen, Topology optimization using a mixed formulation: An alternative way to solve pressure load problems. Comput. Methods Appl. Mech. Eng. 196 (2007) 1874-1889.

[31] J. Sikolowski and J.-P. Zolésio, Introduction to shape optimization, in Shape sensitivity analysis, Springer (1992).

[32] M.Y. Wang and S. Zhou, Synthesis of shape and topology of multi-material structures with a phase-field method. J. ComputerAided Mater. Des. 11 (2004) 117-138.

[33] M.Y. Wang, X. Wang and D. Guo, A level set method for structural topology optimization. Comput. Methods Appl. Mech. Eng. 192 (2003) 227-246.

[34] M.Y. Wang, S. Zhou and H. Ding, Nonlinear diffusions in topology optimization. Struct. Multidisc. Optim. 28 (2004) $262-276$.

[35] P. Wei and M.Y. Wang, Piecewise constant level set method for structural topology optimization. Int. J. Numer. Methods Eng. 78 (2009) 379-402.

[36] Q. Xia and M.Y. Wang, Simultaneous optimization of the material properties and the topology of functionally graded structures. Comput. Aided Des. 40 (2008) 660-675.

[37] S. Zhou and M.Y. Wang, Multimaterial structural topology optimization with a generalized Cahn-Hilliard model of multiphase transition. Struct. Multidisc. Optim. 33 (2007) 89-111. 